\title{
ON CERTAIN CONFORMALLY INVARIANT SYSTEMS OF DIFFERENTIAL EQUATIONS II: FURTHER STUDY OF TYPE A SYSTEMS
}

By

\author{
Anthony C. KABLE
}

\begin{abstract}
Previously, several families of systems of differential equations that generalize the Heisenberg Laplacian equations were introduced. The study of one of these families is continued here. It is shown that the systems in this family are free of integrability conditions provided that a parameter appearing in the system avoids a certain set of bad values, which is explicitly determined. Properties of polynomial solutions to the systems are investigated and special polynomial solutions involving terminating Lauricella hypergeometric series are given in some cases.
\end{abstract}

\section{Introduction}

The Heisenberg Laplacians, introduced by Folland and Stein [2] in their study of the Kohn Laplacian and the $\bar{\partial}_{b}$ complex on the Heisenberg group, have subsequently attracted a great deal of attention. In addition to work from the perspective of analysis of partial differential equations, these operators have also been considered from the special-functions point of view [3], and from the point of view of Lie theory [8]. The analogy between analysis on the Heisenberg group and analysis on Euclidean spaces suggests that other interesting operators might be obtained by varying the signature and, indeed, the resulting operators, which one might reasonably call Heisenberg wave operators, have also received some attention. From the Lie-theory perspective, this list of Heisenberg operators is still incomplete. In that optic, varying the signature amounts to varying the real form of the conformal group of the operator. In addition to the special unitary groups

2010 Mathematics Subject Classification: Primary 35R03; Secondary 35C11, 33C65, 22E25.

Key words and phrases: Heisenberg Laplacian, Lauricella hypergeometric polynomial, systems of differential equations on nilpotent groups, module of polynomial solutions.

Received September 4, 2014. 
$\mathrm{SU}(p, q)$, the special linear group $\operatorname{SL}(n, \mathbf{R})$ also belongs on this list of real forms, and there are operators corresponding to this real form also. The author named these Heisenberg ultrahyperbolic operators, by analogy with the famous Euclidean ultrahyperbolic operator studied by John [4], and investigated certain aspects of these operators $[5,6]$.

Still taking the point of view of Lie theory, one might ask for an intrinsic construction of the Heisenberg Laplacians and their relatives from the associated conformal groups $\mathrm{SU}(p, q)$ and $\operatorname{SL}(n, \mathbf{R})$. Given this, one might then ask whether this construction can be generalized to yield other families of operators with analogous properties. The author took up this question in [7]. The conclusion of this work was that there is such an intrinsic construction, that it depends on some facts about the root system of the Lie algebra $\mathfrak{s l}(n, \mathbf{C})$, and that it can be substantially generalized provided that one is prepared to accept a system of several operators in place of a single operator. The resulting construction yields numerous systems of operators with conformal groups that are special linear or special unitary groups (these are referred to as type A), several systems with conformal groups that are special orthogonal groups (type D), and a system with conformal group the exceptional simple Lie group $\mathrm{E}_{6}$ (type $\mathrm{E}_{6}$ ). Like the Heisenberg Laplacians, each of these systems depends upon a single parameter, which we denote by $z$ rather than the conventional $\alpha$. Each system has its home on a nilpotent Lie group of class 2, which plays the role of the Heisenberg group in the original example.

The purpose of the current work is to continue the study of the systems of type A that was begun in [7]. The properties that we consider are algebraic in nature and so insensitive to the real form. For this reason, we work exclusively with the split real form, whose conformal group is a special linear group. The systems of type $\mathrm{A}$ are described by a triple $(a, b, c)$ of positive natural numbers (in addition to the parameter $z$ ). There are $a c$ operators in the system and they live on the nilpotent Lie group

$$
N=\operatorname{Mat}(a, b) \oplus \operatorname{Mat}(b, c) \oplus \operatorname{Mat}(a, c)
$$

with the operation

$$
(X, Y, T)\left(X^{\prime}, Y^{\prime}, T^{\prime}\right)=\left(X+X^{\prime}, Y+Y^{\prime}, T+T^{\prime}+X Y^{\prime}\right) .
$$

Here $\operatorname{Mat}(m, n)$ denotes the space of $m$-by- $n$ matrices. The center of $N$ is $Z(N)=$ $\{0\} \oplus\{0\} \oplus \operatorname{Mat}(a, c)$ and the center is complemented by the subspace $V=$ $\operatorname{Mat}(a, b) \oplus \operatorname{Mat}(b, c) \oplus\{0\}$, which is, of course, not a subgroup. The Heisenberg case arises when $a=c=1$, and it is only in this case that there is a single operator in the system. 
To explain the context for the first main result, recall that in the theory of the Heisenberg Laplacian one obtains good properties (for example, local solvability) provided that the parameter $z$ does not lie in a discrete set of bad values. The precise set of bad values varies from property to property, but is generally the union of one or more arithmetic progressions. The property we focus on in the first result is that a solution to the system is determined by its restriction to the space $V$ defined above. For reasons that may not presently be obvious, we think of the coordinates on $Z(N)$ as times, so this property may be rephrased to say that a solution to the system is determined by its initial values. We showed in [7, Theorem 4.3] and the subsequent discussion that this property holds for formal power series solutions if and only if $z \notin(b / 2)+\sigma(F)$, where $\sigma(F)$ is the spectrum of a certain operator. This spectrum was determined for each of the systems constructed in [7], except for the system of type A when $b<a$. This case presents a degeneracy that blocks the method used in [7] to determine $\sigma(F)$ when $b \geq a$. Our first main result, Theorem 2.1, is the determination of $\sigma(F)$ in the remaining case, so that this particular set of bad values becomes completely known. We note that $\sigma(F)$ turns out always to be an arithmetic progression in type $\mathrm{A}$; this is not so in types $\mathrm{D}$ and $\mathrm{E}_{6}$.

The transition from a single differential equation to a system of several equations introduces a new issue, that of integrability conditions. The second main result of this work, contained in Theorems 6.4 and 6.5, is that the systems of type A are free of integrability conditions provided that the parameter $z$ does not lie in the bad set $(b / 2)+\sigma(F)$.

From the perspective of Lie theory, there are two classes of solutions to the systems we consider that are of immediate interest, namely the polynomial solutions and the $K$-finite solutions. The former are insensitive to the real form that is chosen and afford interesting modules for the complexified Lie algebra of the conformal group. The latter depend essentially on the real form and lead to the construction of admissible representations for the conformal group itself. We focus here on polynomial solutions when $z$ does not belong to the bad set. The results described in the last two paragraphs imply that given a polynomial $\psi$ on $V$ there is a unique polynomial solution $\Phi$ to the system on $N$ such that $\Phi$ restricts to $\psi$ on $V$. In the Heisenberg case, it is possible to describe these polynomial solutions explicitly in terms of Jacobi polynomials [3], and hence ultimately in terms of the Gaussian hypergeometric series. A more structural but less explicit description of the polynomial solutions in the Heisenberg case was given by Korányi [8]. His result is analogous to Maxwell's well-known description of the harmonic polynomials on Euclidean space and states that a certain vector in 
the module of polynomial solutions is a cyclic vector for the module, so that all polynomial solutions may be obtained by applying the Lie algebra of the conformal group repeatedly to this solution. The author later refined this result somewhat [6] by showing that the module of polynomial solutions is irreducible for most good values of $z$, so that every non-zero vector is a cyclic vector, and also determining the structure of the module for the remaining values of $z$ that were disallowed in Korányi's result. For the general system of type A the structure of the polynomial solutions is much more complicated. It is presently unclear whether an explicit description of these solutions is going to be possible, although it does seem likely that the structure of the module of polynomial solutions can be understood sufficiently well to yield a Korányi-style result as a by-product. For the moment, we are able to exhibit some explicit polynomial solutions to systems of type A in certain cases that directly generalize the known solutions in the Heisenberg case. These are given in Theorems 8.1 and 8.3. They involve terminating Lauricella $F_{D}$ hypergeometric series, which reduce to terminating Gaussian hypergeometric series in the Heisenberg case.

In Section 4 we explain the relation between the form of the systems of type A that we use here and the more usual form in which the equation is written in the Heisenberg case. The difference between the two amounts to a change of coordinates. Although this may seem relatively trivial, choosing the coordinates we employ here has the result that the order of the equations drops from two to one along the center of $N$. This is very convenient because it allows the system to be rewritten in evolutionary form, albeit at the cost of introducing operators more general than differential operators into the picture. In Section 5 we present another useful coordinate change. This one relates the system of type A with parameters $(a, b, c)$ and $z$ to the system of type A with parameters $(c, b, a)$ and $-z$. It is later used in the discussion of explicit polynomial solutions in Section 8 .

\section{Review and Completion of Earlier Results}

We begin by establishing the setting necessary to describe the systems of differential equations that we shall study. Let $l \geq 2$ and $G=\operatorname{SL}(l+1)$. Choose $a, b \geq 1$ such that $a+b \leq l$ and set $c=l-a-b+1$. Let $N$ be the subgroup of $G$ consisting of all upper-triangular matrices of the form

$$
x=\left(\begin{array}{ccc}
I_{a} & X & T \\
0 & I_{b} & Y \\
0 & 0 & I_{c}
\end{array}\right),
$$


where $X \in \operatorname{Mat}(a, b), Y \in \operatorname{Mat}(b, c)$, and $T \in \operatorname{Mat}(a, c)$ are matrices of the indicated sizes. We use the entries in $x$ that lie in the matrices $X, Y$, and $T$ as coordinates on $N$. In order to make it easy to distinguish these coordinates, we introduce the abbreviations

$$
\begin{array}{ll}
\mu(i, j)=(i, a+j) & \text { for } 1 \leq i \leq a, 1 \leq j \leq b, \\
v(j, k)=(a+j, a+b+k) & \text { for } 1 \leq j \leq b, 1 \leq k \leq c, \\
\zeta(i, k)=(i, a+b+k) & \text { for } 1 \leq i \leq a, 1 \leq k \leq c .
\end{array}
$$

With these abbreviations, $X=\left[x_{\mu(i, j)}\right], Y=\left[x_{v(j, k)}\right]$, and $T=\left[x_{\zeta(i, k)}\right]$. For comparison with [7], note that we may identify $\mu(i, j)$ with the root $e_{i}-e_{a+j}$ in the standard model for the root system of $G$, and similarly with $v(j, k)$ and $\zeta(i, k)$. The group $N$ is the unipotent radical of the standard parabolic subgroup $Q$ of $G$ corresponding to the deleted Dynkin diagram

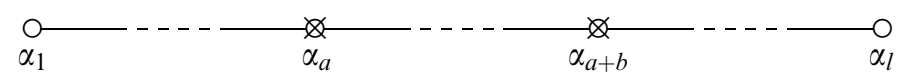

with the usual positive system and numbering of the simple roots. In keeping with the notation introduced in [7], let $z_{0}=b / 2$ be half the distance between the two deleted simple roots. In addition, let

$$
\begin{aligned}
& R_{12}=\{\mu(i, j) \mid 1 \leq i \leq a, 1 \leq j \leq b\}, \\
& R_{23}=\{v(j, k) \mid 1 \leq j \leq b, 1 \leq k \leq c\}, \\
& R_{13}=\{\zeta(i, k) \mid 1 \leq i \leq a, 1 \leq k \leq c\}
\end{aligned}
$$

be the sets of roots associated with the submatrices $X, Y$, and $T$ of $x$. For each coordinate $x_{\gamma}$ on $N$, let $\partial_{\gamma}$ be the corresponding partial derivative.

We are now ready to describe the system of partial differential operators that we wish to study. For $1 \leq i \leq a$ and $1 \leq k \leq c$, let

$$
\Delta_{\zeta(i, k)}=\sum_{j=1}^{b} \partial_{\mu(i, j)} \partial_{v(j, k)} .
$$

For $1 \leq i, p \leq a$ and $1 \leq k, r \leq c$, let

$$
F_{\zeta(i, k), \zeta(p, r)}=\delta_{k r} \sum_{j=1}^{b} x_{\mu(p, j)} \partial_{\mu(i, j)},
$$


where $\delta$ is the Kronecker delta. For $\zeta \in R_{13}$, let

$$
\Omega_{\zeta}=\Delta_{\zeta}-\left(z-z_{0}\right) \partial_{\zeta}+\sum_{\xi \in R_{13}} F_{\zeta, \xi} \partial_{\xi}
$$

where $z$ is a parameter. The system in question is comprised of all the operators $\Omega_{\zeta}$ with $\zeta \in R_{13}$. This is a system of ac differential equations on $N$.

The system $\Omega_{\zeta}, \zeta \in R_{13}$, was first constructed in [7] in a more general context. The general form of the systems constructed in that work may be found in [7, Proposition 4.2] and the explicit forms of the operators $\Delta$ and $F$ in the present case are given in [7, Section 5.1]. However, the reader who consults [7] will notice some sign differences between the expressions given there and those given above. In [7], the proofs were written, as far as possible, in a uniform way. This necessitated a uniform choice of model for the Lie algebras underlying the construction, and this model does not necessarily specialize to the most convenient model in any particular instance. In particular, the root vectors in $\mathfrak{s l}(l+1)$ used in [7] were the negatives of those that are usually chosen. Now that the focus is on a single family of algebras, it was thought best to change coordinates to restore the conventional choices. This amounts to the transformation $x_{\gamma} \mapsto-x_{\gamma}$ and explains the sign changes noted above.

We may express the condition $\Omega_{\zeta} \bullet \Phi=0, \zeta \in R_{13}$, in vector form as

$$
\left[\Delta_{\zeta} \bullet \Phi\right]=\left(\left(z-z_{0}\right) I-F\right)\left[\partial_{\zeta} \bullet \Phi\right],
$$

where $F=\left[F_{\zeta, \xi}\right]$ is an $R_{13}$-by- $R_{13}$ matrix of differential operators. It is desirable to rewrite this in evolutionary form as

$$
\left[\partial_{\zeta} \bullet \Phi\right]=\left(\left(z-z_{0}\right) I-F\right)^{-1}\left[\Delta_{\zeta} \bullet \Phi\right]
$$

and this will be possible provided that $z-z_{0} \notin \sigma(F)$, where $\sigma(F)$ denotes the spectrum of $F$. Of course, the appropriate definition of the spectrum will depend on the context. For our present purposes, we consider the action of $F$ on the space $\mathbf{C}\left[x_{\mu}\right] \otimes \mathbf{C}^{R_{13}}$ of $R_{13}$-tuples of polynomials in the variables $x_{\mu}$, $\mu \in R_{12}$. Although this space has infinite dimension, the action is locally finite and the spectrum $\sigma(F)$ is simply the set of eigenvalues of $F$. In [7, Section 5], this set was determined in all cases except for the systems in type A with $1<b<a$. We now wish to complete this result by determining $\sigma(F)$ in the remaining cases. For clarity, note that we take the natural numbers $\mathbf{N}$ to include 0 . 
THEOREM 2.1. We have

$$
\sigma(F)= \begin{cases}-(a-1)+\mathbf{N} & \text { if } b \geq a, \\ -b+\mathbf{N} & \text { if } b<a .\end{cases}
$$

Proof. The evaluation of $\sigma(F)$ when $b \geq a$ is given in [7, Theorem 5.4]. We henceforth assume that $b<a$. Since the entries in $F$ are differential operators in the variables $x_{\mu}$ with $\mu \in R_{12}$, we shall use the abbreviations $x_{\mu(i, j)}=x_{i, j}$ and $\partial_{\mu(i, j)}=\partial_{i, j}$ for the remainder of the proof. With this abbreviation, we have

$$
F_{\zeta(i, k), \zeta(p, r)}=\delta_{k r} \sum_{j=1}^{b} x_{p, j} \partial_{i, j} .
$$

It follows that if we order the $\zeta(i, k)$ suitably then the matrix $F$ becomes a block sum

$$
F=P \boxplus P \boxplus \cdots \boxplus P
$$

where $P$ is the $a$-by- $a$ matrix of differential operators with entries

$$
P_{i, p}=\sum_{j=1}^{b} x_{p, j} \partial_{i, j}
$$

and there are $c$ blocks in the block sum. Thus it suffices to determine the spectrum of $P$ acting on the space $\mathbf{C}\left[x_{i, j}\right] \otimes \mathbf{C}^{a}$ of $a$-tuples of polynomials in the variables $x_{i, j}$ with $1 \leq i \leq a$ and $1 \leq j \leq b$. Next we wish to introduce a group action on $\mathbf{C}\left[x_{i, j}\right] \otimes \mathbf{C}^{a}$ with respect to which $P$ acts equivariantly.

The group $H=\mathrm{GL}(a) \times \mathrm{GL}(b)$ acts by automorphisms on the polynomial algebra $\mathbf{C}\left[x_{i, j}\right]$. For $g \in \mathrm{GL}(a)$ we have

$$
g x_{i, j}=\sum_{p=1}^{a} g_{p, i} x_{p, j}
$$

and for $h \in \mathrm{GL}(b)$ we have

$$
h x_{i, j}=\sum_{q=1}^{b} x_{i, q} h_{q, j} .
$$

The induced actions on the partial derivatives are

$$
g \partial_{i, j}=\sum_{p=1}^{a} \bar{g}_{i, p} \partial_{p, j}
$$


and

$$
h \partial_{i, j}=\sum_{q=1}^{b} \partial_{i, q} \bar{h}_{j, q}
$$

where the bar denotes the entries in the inverse matrix. These extend to an action of $H$ on the algebra $\mathbf{C}\left[\partial_{i, j}\right]$ by automorphisms. This action is such that the evaluation map $(D, \psi) \mapsto D \bullet \psi$ from $\mathbf{C}\left[\partial_{i, j}\right] \times \mathbf{C}\left[x_{i, j}\right]$ to $\mathbf{C}\left[x_{i, j}\right]$ is $H$-equivariant. The same is true of the multiplication operators associated to elements of $\mathbf{C}\left[x_{i, j}\right]$, and hence the above actions of $H$ extend to an action on the Weyl algebra $\mathbf{C}\left[x_{i, j}, \partial_{i, j}\right]$ such that the evaluation map $\mathbf{C}\left[x_{i, j}, \partial_{i, j}\right] \times \mathbf{C}\left[x_{i, j}\right] \rightarrow \mathbf{C}\left[x_{i, j}\right]$ is $H$ equivariant.

For $g \in \mathrm{GL}(a)$, we have

$$
g^{-1} P_{i, p}=\sum_{s, t=1}^{a} \bar{g}_{s, p} g_{i, t} P_{t, s} .
$$

Let $\mathrm{GL}(a)$ act on $\mathbf{C}^{a}$ by the standard action, so that

$$
g e_{m}=\sum_{v=1}^{a} g_{v, m} e_{v}
$$

and give $\mathbf{C}\left[x_{i, j}\right] \otimes \mathbf{C}^{a}$ the resulting tensor product action. For

$$
\varphi=\sum_{m=1}^{a} \varphi_{m} \otimes e_{m} \in \mathbf{C}\left[x_{i, j}\right] \otimes \mathbf{C}^{a}
$$

we have

$$
P \bullet \varphi=\sum_{m, n=1}^{a}\left(P_{m, n} \bullet \varphi_{n}\right) \otimes e_{m} .
$$

This gives us all the ingredients necessary to establish the $\operatorname{GL}(a)$-equivariance of $P$. With $\varphi$ as above, we have

$$
\begin{aligned}
P \bullet(g \varphi) & =P \bullet \sum_{m=1}^{a} g \varphi_{m} \otimes g e_{m} \\
& =P \bullet \sum_{m, v=1}^{a} g_{v, m}\left(g \varphi_{m}\right) \otimes e_{v}
\end{aligned}
$$




$$
\begin{aligned}
& =\sum_{m, v, n=1}^{a} P_{v, n} \bullet\left(g_{n, m}\left(g \varphi_{m}\right)\right) \otimes e_{v} \\
& =\sum_{m, v, n=1}^{a} g_{n, m} P_{v, n} \bullet\left(g \varphi_{m}\right) \otimes e_{v} \\
& =g \sum_{m, v, n=1}^{a} g_{n, m}\left(g^{-1} P_{v, n}\right) \bullet \varphi_{m} \otimes g^{-1} e_{v} \\
& =g \sum_{m, v, n, s, t, u=1}^{a} \bar{g}_{u, v} g_{n, m} \bar{g}_{s, n} g_{v, t}\left(P_{t, s} \bullet \varphi_{m}\right) \otimes e_{u} \\
& =g \sum_{m, s, t, u=1}^{a} \delta_{s m} \delta_{u t}\left(P_{t, s} \bullet \varphi_{m}\right) \otimes e_{u} \\
& =g \sum_{m, u=1}^{a}\left(P_{u, m} \bullet \varphi_{m}\right) \otimes e_{u} \\
& =g(P \bullet \varphi) .
\end{aligned}
$$

That is, $P$ acts $\mathrm{GL}(a)$-equivariantly on $\mathbf{C}\left[x_{i, j}\right] \otimes \mathbf{C}^{a}$.

For $h \in \mathrm{GL}(b)$ we have

$$
h P_{i, p}=P_{i, p}
$$

We make $\mathrm{GL}(b)$ act on $\mathbf{C}\left[x_{i, j}\right] \otimes \mathbf{C}^{a}$ by giving the second factor the trivial action. It follows that we have

$$
P \bullet(h \varphi)=h(P \bullet \varphi)
$$

for all $h \in \mathrm{GL}(b)$ and $\varphi \in \mathbf{C}\left[x_{i, j}\right] \otimes \mathbf{C}^{a}$. That is, $P$ also acts $\operatorname{GL}(b)$-equivariantly on $\mathbf{C}\left[x_{i, j}\right] \otimes \mathbf{C}^{a}$.

The next step is to recall the decomposition of $\mathbf{C}\left[x_{i, j}\right] \otimes \mathbf{C}^{a}$ as an $H$-module and describe the highest weight vectors associated to this decomposition. If $D$ is a Young diagram of depth at most $b$ then we write $\rho_{a}(D)$ and $\rho_{b}(D)$ for the polynomial representations of $\operatorname{GL}(a)$ and $\operatorname{GL}(b)$, respectively, corresponding to $D$. It follows from the First Fundamental Theorem of Invariant Theory and Pieri's Rule that

$$
\mathbf{C}\left[x_{i, j}\right] \otimes \mathbf{C}^{a} \cong \underset{\left(D_{1}, D_{2}\right)}{\bigoplus_{a}} \rho_{a}\left(D_{1}\right) \otimes \rho_{b}\left(D_{2}\right)
$$


as $H$-modules, where the sum is over pairs of Young diagrams $\left(D_{1}, D_{2}\right)$ such that the depth of $D_{2}$ is at most $b$ and $D_{1}$ may be obtained from $D_{2}$ by adding a single box. If $\left(D_{1}, D_{2}\right)$ is such a pair then it is unambiguous which box has been added to $D_{2}$ to obtain $D_{1}$. The pair $\left(D_{1}, D_{2}\right)$ can be conveniently displayed by drawing the Young diagram for $D_{1}$ with the added box shaded. We define $\left(D_{1}, D_{2}\right)^{\prime}$ to be the Young diagram obtained from $D_{1}$ by deleting the added box and all boxes above it in the diagram, and then pushing detached boxes to the left if necessary. For example, if

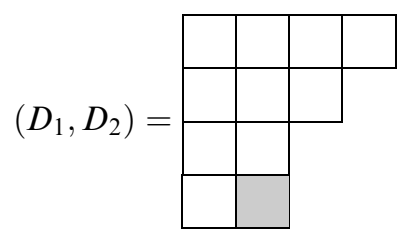

then

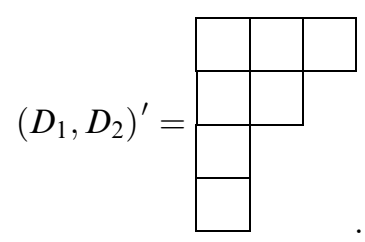

In order to describe the highest weight vectors in $\mathbf{C}\left[x_{i, j}\right] \otimes \mathbf{C}^{a}$, we begin by defining

$$
\Theta_{q}=\operatorname{det}\left[\begin{array}{cccc}
x_{1,1} & x_{1,2} & \cdots & x_{1, q} \\
x_{2,1} & x_{2,2} & \cdots & x_{2, q} \\
\vdots & \vdots & & \vdots \\
x_{q, 1} & x_{q, 2} & \cdots & x_{q, q}
\end{array}\right] \in \mathbf{C}\left[x_{i, j}\right]
$$

for $1 \leq q \leq b$ and

$$
\Xi_{q}=\operatorname{det}\left[\begin{array}{ccccc}
x_{1,1} & x_{1,2} & \cdots & x_{1, q-1} & \otimes e_{1} \\
x_{2,1} & x_{2,2} & \cdots & x_{2, q-1} & \otimes e_{2} \\
\vdots & \vdots & & \vdots & \vdots \\
x_{q, 1} & x_{q, 2} & \cdots & x_{q, q-1} & \otimes e_{q}
\end{array}\right] \in \mathbf{C}\left[x_{i, j}\right] \otimes \mathbf{C}^{a}
$$

for $1 \leq q \leq b+1$. The determinant defining $\Xi_{q}$ is to be expanded by minors along the last column and, taking the empty determinant to equal $1, \Xi_{1}=1 \otimes e_{1}$. 
Let us fix the standard maximal tori and positive systems in $\operatorname{GL}(a)$ and $\operatorname{GL}(b)$. Then $\Theta_{q}$ and $\Xi_{q}$ are evidently highest weight vectors for the action of $H$ on $\mathbf{C}\left[x_{i, j}\right]$ and $\mathbf{C}\left[x_{i, j}\right] \otimes \mathbf{C}^{a}$, respectively. The weight of $\Theta_{q}$ is

$$
\varpi\left(\Theta_{q}\right)=((\underbrace{1, \ldots, 1}_{q}, \underbrace{0, \ldots, 0}_{a-q}),(\underbrace{1, \ldots, 1}_{q}, \underbrace{0, \ldots, 0}_{b-q}))
$$

and the weight of $\Xi_{q}$ is

$$
\varpi\left(\Xi_{q}\right)=((\underbrace{1, \ldots, 1}_{q}, \underbrace{0, \ldots, 0}_{a-q}),(\underbrace{1, \ldots, 1}_{q-1}, \underbrace{0, \ldots, 0}_{b-q+1})) .
$$

If $D$ is a Young diagram of depth at most $b$ and the corresponding partition is $\pi(D)=\left(m_{1}, m_{2}, \ldots, m_{b}\right)$ then we define

$$
\Theta(D)=\Theta_{1}^{m_{1}-m_{2}} \Theta_{2}^{m_{2}-m_{3}} \cdots \Theta_{b-1}^{m_{b-1}-m_{b}} \Theta_{b}^{m_{b}} .
$$

This is a highest weight vector in $\mathbf{C}\left[x_{i, j}\right]$ of weight

$$
\varpi(\Theta(D))=((\pi(D), \underbrace{0, \ldots, 0}_{a-b}), \pi(D)) .
$$

Finally, if $\left(D_{1}, D_{2}\right)$ is a pair of Young diagrams of the type occurring in the decomposition of $\mathbf{C}\left[x_{i, j}\right] \otimes \mathbf{C}^{a}$ and the extra box in $D_{1}$ occurs in the $q^{\text {th }}$ row then we define

$$
v\left(D_{1}, D_{2}\right)=\Theta\left(\left(D_{1}, D_{2}\right)^{\prime}\right) \Xi_{q}
$$

This is a highest weight vector in $\mathbf{C}\left[x_{i, j}\right] \otimes \mathbf{C}^{a}$ with the weight corresponding to the component $\rho_{a}\left(D_{1}\right) \otimes \rho_{b}\left(D_{2}\right)$ in the decomposition of $\mathbf{C}\left[x_{i, j}\right] \otimes \mathbf{C}^{a}$. Thus we have described a complete set of highest weight vector in $\mathbf{C}\left[x_{i, j}\right] \otimes \mathbf{C}^{a}$.

Since the map $P$ is $H$-equivariant and $\mathbf{C}\left[x_{i, j}\right] \otimes \mathbf{C}^{a}$ is multiplicity free as an $H$-module, $P$ acts on each component $\rho_{a}\left(D_{1}\right) \otimes \rho_{b}\left(D_{2}\right)$ by a scalar $\lambda\left(D_{1}, D_{2}\right)$. The spectrum $\sigma(P)$ of $P$ on $\mathbf{C}\left[x_{i, j}\right] \otimes \mathbf{C}^{a}$ is precisely the set of the $\lambda\left(D_{1}, D_{2}\right)$, and so it remains to determine them. We have

$$
P \bullet v\left(D_{1}, D_{2}\right)=\lambda\left(D_{1}, D_{2}\right) v\left(D_{1}, D_{2}\right)
$$

and this will serve to identify $\lambda\left(D_{1}, D_{2}\right)$ if we can compute $P \bullet v\left(D_{1}, D_{2}\right)$. 
We have

$$
\begin{aligned}
P_{i, p} & =\sum_{j=1}^{b} x_{p, j} \partial_{i, j} \\
& =\sum_{j=1}^{b}\left(-\delta_{i p}+\partial_{i, j} x_{p, j}\right) \\
& =-b \delta_{i p}+\sum_{j=1}^{b} \partial_{i, j} x_{p, j}
\end{aligned}
$$

from which it follows that

$$
P=-b I_{a}+M N
$$

where

$$
M=\left[\begin{array}{cccc}
\partial_{1,1} & \partial_{1,2} & \cdots & \partial_{1, b} \\
\partial_{2,1} & \partial_{2,2} & \cdots & \partial_{2, b} \\
\vdots & \vdots & & \vdots \\
\partial_{a, 1} & \partial_{a, 2} & \cdots & \partial_{a, b}
\end{array}\right]
$$

and

$$
N=\left[\begin{array}{cccc}
x_{1,1} & x_{2,1} & \cdots & x_{a, 1} \\
x_{1,2} & x_{2,2} & \cdots & x_{a, 2} \\
\vdots & \vdots & & \vdots \\
x_{1, b} & x_{2, b} & \cdots & x_{a, b}
\end{array}\right] .
$$

Let $\left(D_{1}, D_{2}\right)$ be a pair of Young diagrams, as above, and set $D=\left(D_{1}, D_{2}\right)^{\prime}$. Suppose that the extra box in $D_{1}$ occurs in the $q^{\text {th }}$ row. Let $C_{i}$ be the signed cofactor of $e_{i}$ in $\Xi_{q}$ so

$$
\Xi_{q}=\sum_{i=1}^{q} C_{i} \otimes e_{i} .
$$

For later reference, note that $C_{q}=\Theta_{q-1}$ provided that we define $\Theta_{0}=1$. We have

$$
N e_{i}=\sum_{j=1}^{b} x_{i, j} \otimes e_{j}
$$


and so

$$
N \Xi_{q}=\sum_{i=1}^{q} \sum_{j=1}^{b} x_{i, j} C_{i} \otimes e_{j}
$$

We have

$$
M e_{j}=\sum_{k=1}^{a} \partial_{k, j} e_{k}
$$

and so

$$
\begin{aligned}
M N\left(\Theta(D) \Xi_{q}\right)= & \sum_{k=1}^{a} \sum_{i=1}^{q} \sum_{j=1}^{b} \partial_{k, j} \bullet\left(x_{i, j} \Theta(D) C_{i}\right) \otimes e_{k} \\
= & \sum_{i=1}^{q} \sum_{j=1}^{b} \Theta(D) C_{i} \otimes e_{i}+\sum_{k=1}^{a} \sum_{i=1}^{q} \sum_{j=1}^{b} x_{i, j}\left(\partial_{k, j} \bullet \Theta(D)\right) C_{i} \otimes e_{k} \\
& +\sum_{k=1}^{a} \sum_{i=1}^{q} \sum_{j=1}^{b} x_{i, j} \Theta(D)\left(\partial_{k, j} \bullet C_{i}\right) \otimes e_{k} .
\end{aligned}
$$

Since we already know that $M N\left(\Theta(D) \Xi_{q}\right)$ is a multiple of $\Theta(D) \Xi_{q}$, we need only evaluate a single non-zero component in $M N\left(\Theta(D) \Xi_{q}\right)$ in order to determine $\lambda\left(D_{1}, D_{2}\right)$. We choose the $q^{\text {th }}$ component, which is a sum of three terms. The first is

$$
\sum_{j=1}^{b} \Theta(D) C_{q}=b \Theta(D) C_{q}=b \Theta(D) \Theta_{q-1}
$$

The second is

$$
\sum_{i=1}^{q} \sum_{j=1}^{b} x_{i, j}\left(\partial_{q, j} \bullet \Theta(D)\right) C_{i}=\sum_{i=1}^{q} C_{i} \sum_{j=1}^{b} x_{i, j} \partial_{q, j} \bullet \Theta(D)
$$

The differential operator applied to $\Theta(D)$ in this sum obeys the Leibniz Rule and so we can deduce the value of the sum by first evaluating

$$
\sum_{j=1}^{b} x_{i, j} \partial_{q, j} \bullet \Theta_{n}
$$


If $n<q$ then this expression is 0 , because $\partial_{q, j} \bullet \Theta_{n}=0$ for all $1 \leq j \leq b$. Suppose that $n \geq q$. Then

$$
\partial_{q, j} \bullet \Theta_{n}=(-1)^{q+j} \Theta_{n}[q, j],
$$

where $\Theta_{n}[q, j]$ denotes the cofactor corresponding to the $(q, j)$-entry in the matrix defining $\Theta_{n}$, if $1 \leq j \leq n$, and $\partial_{q, j} \bullet \Theta_{n}=0$ otherwise. Thus

$$
\sum_{j=1}^{b} x_{i, j} \partial_{q, j} \bullet \Theta_{n}= \begin{cases}0 & \text { if } n<q \text { or } i \neq q \\ \Theta_{n} & \text { if } n \geq q \text { and } i=q\end{cases}
$$

since when $n \geq q$ the expression is the determinant of the matrix obtained by replacing the $q^{\text {th }}$ row in the determinant defining $\Theta_{n}$ by the $i^{\text {th }}$ row. Let $\pi(D)=$ $\left(m_{1}, \ldots, m_{b}\right)$ be the partition associated to $D$, padded with zeros if necessary. The evaluation we have just completed, together with the Leibniz Rule, implies that

$$
\sum_{j=1}^{b} x_{i, j} \partial_{q, j} \bullet \Theta(D)=0
$$

if $i \neq q$. If $i=q$ then we instead obtain

$$
\begin{aligned}
\sum_{j=1}^{b} x_{q, j} \partial_{q, j} \bullet \Theta(D) & =\left[\left(m_{q}-m_{q+1}\right)+\left(m_{q+1}-m_{q+2}\right)+\cdots+m_{b}\right] \Theta(D) \\
& =m_{q} \Theta(D) .
\end{aligned}
$$

Thus the second term in the original sum is

$$
\sum_{i=1}^{q} \sum_{j=1}^{b} x_{i, j}\left(\partial_{q, j} \bullet \Theta(D)\right) C_{i}=C_{q} m_{q} \Theta(D)=m_{q} \Theta(D) \Theta_{q-1} .
$$

It remains to evaluate the third term, which is

$$
\sum_{i=1}^{q} \sum_{j=1}^{b} x_{i, j} \Theta(D)\left(\partial_{q, j} \bullet C_{i}\right)
$$

If $i=q$ then $\partial_{q, j} \bullet C_{i}=0$ for all $j$ and so this sum is equal to

$$
\Theta(D) \sum_{i=1}^{q-1} \sum_{j=1}^{b} x_{i, j} \partial_{q, j} \bullet C_{i}
$$


Let $R$ denote the matrix whose determinant defines $\Xi_{q}$. Then the inner sum is

$$
\begin{aligned}
\sum_{j=1}^{b} x_{i, j} \partial_{q, j} \bullet C_{i} & =\sum_{j=1}^{q-1} x_{i, j}(-1)^{i+q} \partial_{q, j} \bullet \operatorname{det} R[i, q] \\
& =\sum_{j=1}^{q-1} x_{i, j}(-1)^{i+q}(-1)^{q+j-1} \operatorname{det} R[i, q][q-1, j] \\
& =-\sum_{j=1}^{q-1}(-1)^{i+j} x_{i, j} \Theta_{q-1}[i, j] \\
& =-\Theta_{q-1},
\end{aligned}
$$

where $R[i, q]$ denotes the $(i, q)$-minor matrix in $R, R[i, q][q-1, j]$ the $(q-1, j)$ minor matrix in $R[i, q]$, and $\Theta_{q-1}[i, j]$ denotes the $(i, j)$-cofactor in the determinant of the matrix defining $\Theta_{q-1}$. Thus the third term is

$$
-\Theta(D) \sum_{i=1}^{q-1} \Theta_{q-1}=(1-q) \Theta(D) \Theta_{q-1} .
$$

By combining these evaluations, we obtain

$$
\operatorname{MN}\left(\Theta(D) \Xi_{q}\right)=\left(b+m_{q}+1-q\right) \Theta(D) \Xi_{q}
$$

and so

$$
P \bullet\left(\Theta(D) \Xi_{q}\right)=\left(1-q+m_{q}\right) \Theta(D) \Xi_{q} .
$$

Note that $m_{q}$, the number of boxes in the $q^{\text {th }}$ row of $D=\left(D_{1}, D_{2}\right)^{\prime}$, is the same as the number of boxes in the $q^{\text {th }}$ row of $D_{2}$. Thus we have

$$
\lambda\left(D_{1}, D_{2}\right)=1-q+m_{q}\left(D_{2}\right)
$$

To complete the proof, we must show that the possible values of $\lambda\left(D_{1}, D_{2}\right)$, as $\left(D_{1}, D_{2}\right)$ varies over all valid pairs of Young diagrams, coincides with the arithmetic progression $-b+\mathbf{N}$. Since $1 \leq q \leq b+1$ and $m_{q}\left(D_{2}\right) \in \mathbf{N}$, the possible values of $\lambda\left(D_{1}, D_{2}\right)$ are all contained in this arithmetic progression. To achieve the value $-r$ with $1 \leq r \leq b$, we may take $D_{2}$ to be the Young diagram with a single box in each of the first $r$ rows, and then obtain $D_{1}$ by adding a single box in the $(r+1)^{\text {st }}$ row. To achieve the value $r$ with $r \in \mathbf{N}$, we may take $D_{2}$ to 
be the Young diagram with $r$ boxes in the first row, and then obtain $D_{1}$ from it by adding an additional box in the first row. This verifies that every element of $-b+\mathbf{N}$ is of the form $\lambda\left(D_{1}, D_{2}\right)$ for some valid pair $\left(D_{1}, D_{2}\right)$, as required.

\section{Some Useful Notation and Facts}

For ease of reference, it will be convenient to collect together the definitions of various objects that will occur frequently. We shall also take the opportunity to record various relationships enjoyed by these objects. We define

$$
P_{i, p}=\sum_{j=1}^{b} x_{\mu(p, j)} \partial_{\mu(i, j)}
$$

for $1 \leq i, p \leq a$,

$$
Q_{r, k}=\sum_{j=1}^{b} x_{v(j, r)} \partial_{v(j, k)}
$$

for $1 \leq r, k \leq c$, and

$$
\Delta_{\zeta(i, k)}=\sum_{j=1}^{b} \partial_{\mu(i, j)} \partial_{v(j, k)}
$$

for $1 \leq i \leq a, 1 \leq k \leq c$. We shall call a function $\psi$ harmonic when $\Delta_{\zeta} \bullet \psi=0$ for all $\zeta \in R_{13}$.

LeMma 3.1. We have

$$
\left[P_{i, p}, P_{s, t}\right]=\delta_{i t} P_{s, p}-\delta_{p s} P_{i, t}
$$

and

$$
\left[Q_{r, k}, Q_{s, t}\right]=\delta_{k s} Q_{r, t}-\delta_{t r} Q_{s, k}
$$

Proof. Routine computation.

Lemma 3.2. We have

$$
\left[\Delta_{\zeta(i, k)}, P_{s, p}\right]=\delta_{i p} \Delta_{\zeta(s, k)}
$$


and

$$
\left[\Delta_{\zeta(i, k)}, Q_{r, s}\right]=\delta_{k r} \Delta_{\zeta(i, s)}
$$

Proof. Routine computation.

For $1 \leq i \leq a$ and $1 \leq k \leq c$, we define

$$
\Omega_{\zeta(i, k)}^{(z)}=\Delta_{\zeta(i, k)}-\left(z-z_{0}\right) \partial_{\zeta(i, k)}+\sum_{p=1}^{a} P_{i, p} \partial_{\zeta(p, k)},
$$

where $z_{0}=b / 2$. The $(z)$ is omitted from the notation when the value of the parameter $z$ need not be emphasized. The $\Omega$-system is the system of differential operators $\Omega_{\zeta}^{(z)}, \zeta \in R_{13}$. We define

$$
\varphi_{\zeta(i, k)}=\sum_{j=1}^{b} x_{\mu(i, j)} x_{v(j, k)}
$$

for $1 \leq i \leq a$ and $1 \leq k \leq c$. For parameters $\beta(\zeta), \zeta \in R_{13}$, we define

$$
\varphi^{\beta}=\prod_{\xi \in R_{13}} \varphi_{\xi}^{\beta(\xi)} .
$$

Here, as usual in algebraic treatments of differential equations, $\psi^{\alpha}$ is to be interpreted as a symbol that satisfies the relation

$$
\partial_{\gamma} \bullet \psi^{\alpha}=\alpha \psi^{\alpha} \psi^{-1} \partial_{\gamma} \bullet \psi
$$

for all $\gamma \in R_{12} \cup R_{23} \cup R_{13}$. We may write $\psi^{\alpha-1}$ for $\psi^{\alpha} \psi^{-1}$ when this will not cause confusion.

Lemma 3.3. We have

$$
\partial_{\mu(i, j)} \bullet \varphi^{\beta}=\varphi^{\beta} \sum_{r=1}^{c} \beta(\zeta(i, r)) \varphi_{\zeta(i, r)}^{-1} x_{v(j, r)}
$$

and

$$
\partial_{v(j, k)} \bullet \varphi^{\beta}=\varphi^{\beta} \sum_{p=1}^{a} \beta(\zeta(p, k)) \varphi_{\zeta(p, k)}^{-1} x_{\mu(p, j)} .
$$

Proof. Routine computation. 
Lemma 3.4. We have

$$
P_{i, p} \bullet \varphi^{\beta}=\varphi^{\beta} \sum_{r=1}^{c} \beta(\zeta(i, r)) \varphi_{\zeta(i, r)}^{-1} \varphi_{\zeta(p, r)}
$$

and

$$
Q_{r, k} \bullet \varphi^{\beta}=\varphi^{\beta} \sum_{p=1}^{a} \beta(\zeta(p, k)) \varphi_{\zeta(p, k)}^{-1} \varphi_{\zeta(p, r)}
$$

Proof. Routine computation using Lemma 3.3.

Proposition 3.5. We have

$$
\begin{aligned}
\Delta_{\zeta(i, k)} \bullet\left(\varphi^{\beta} \psi\right)= & \varphi^{\beta} \psi \sum_{p=1}^{a} \sum_{r=1}^{c} \beta(\zeta(i, r)) \beta(\zeta(p, k)) \varphi_{\zeta(i, r)}^{-1} \varphi_{\zeta(p, k)}^{-1} \varphi_{\zeta(p, r)} \\
& +\varphi^{\beta} \sum_{p=1}^{a} \beta(\zeta(p, k)) \varphi_{\zeta(p, k)}^{-1}\left(P_{i, p} \bullet \psi\right) \\
& +\varphi^{\beta} \sum_{r=1}^{c} \beta(\zeta(i, r)) \varphi_{\zeta(i, r)}^{-1}\left(Q_{r, k} \bullet \psi\right) \\
& +(b-1) \beta(\zeta(i, k)) \varphi^{\beta} \varphi_{\zeta(i, k)}^{-1} \psi+\varphi^{\beta} \Delta_{\zeta(i, k)} \bullet \psi .
\end{aligned}
$$

Proof. The formula results from a slightly involved computation. We begin by using Lemma 3.3 to write

$$
\partial_{v(j, k)} \bullet\left(\varphi^{\beta} \psi\right)=\varphi^{\beta} \partial_{v(j, k)} \bullet \psi+\varphi^{\beta} \psi \sum_{p=1}^{a} \beta(\zeta(p, k)) \varphi_{\zeta(p, k)}^{-1} x_{\mu(p, j)}
$$

We then act on this identity by $\partial_{\mu(i, j)}$ and use Lemma 3.3 once again to write

$$
\partial_{\mu(i, j)} \partial_{v(j, k)} \bullet\left(\varphi^{\beta} \psi\right)=1+\cdots+6,
$$

a sum of six terms. Each of these terms must then be summed over $1 \leq j \leq b$. The first term is $1=\varphi^{\beta} \partial_{\mu(i, j)} \partial_{v(j, k)} \bullet \psi$ and these sum to $\varphi^{\beta} \Delta_{\zeta(i, k)} \bullet \psi$. The second is

$$
2=\varphi^{\beta} \sum_{r=1}^{c} \beta(\zeta(i, r)) \varphi_{\zeta(i, r)}^{-1} x_{v(j, r)} \partial_{v(j, k)} \bullet \psi
$$


and these sum to the term involving $Q_{r, k}$. Similarly, the third is

$$
3=\varphi^{\beta} \sum_{p=1}^{a} \beta(\zeta(p, k)) \varphi_{\zeta(p, k)}^{-1} x_{\mu(p, j)} \partial_{\mu(i, j)} \bullet \psi
$$

and these sum to the term involving $P_{i, p}$. The fourth is

$$
4=\varphi^{\beta} \psi\left(\sum_{p=1}^{a} \beta(\zeta(p, k)) \varphi_{\zeta(p, k)}^{-1} x_{\mu(p, j)}\right)\left(\sum_{r=1}^{c} \beta(\zeta(i, r)) \varphi_{\zeta(i, r)}^{-1} x_{v(j, r)}\right)
$$

and these sum to the first term in the statement. The fifth is

$$
5=-\beta(\zeta(i, k)) \varphi^{\beta} \varphi_{\zeta(i, k)}^{-2} x_{\mu(i, j)} x_{v(j, k)} \psi
$$

and these sum to $-\beta(\zeta(i, k)) \varphi^{\beta} \varphi_{\zeta(i, k)}^{-1} \psi$. The sixth is $6=\beta(\zeta(i, k)) \varphi^{\beta} \varphi_{\zeta(i, k)}^{-1} \psi$ and these sum to $b \beta(\zeta(i, k)) \varphi^{\beta} \varphi_{\zeta(i, k)}^{-1} \psi$. By combining the last two sums we complete the evaluation.

\section{Comparison with the Usual Expression for the Heisenberg Ultrahyperbolic Operator}

We have remarked above that the $\Omega$-system is a generalization of the Heisenberg Ultrahyperbolic operator, which belongs to a family of operators that includes the Heisenberg Laplacian operator. The reader familiar with the standard form of the Heisenberg Laplacian operator may be puzzled by this statement, because the Heisenberg Laplacian operator, as it is usually written, has a term involving $\partial^{2} / \partial t^{2}$. In our notation, this is $\partial_{\zeta(1,1)}^{2}$ and no such term appears in the $\Omega$-system. If it did then it would not be possible to express the $\Omega$-system in evolutionary form, as we have done. The solution to this puzzle is that the two forms are related by a change of coordinates. In the standard coordinates, which we refer to as Lie algebra coordinates, the $\Omega$-system has a more symmetrical appearance but second derivatives along the center appear. In the coordinates we are using here, which we refer to as Lie group coordinates, the symmetrical appearance is lost, but so are the second derivatives along the center. This makes the Lie group coordinates preferable for many purposes.

To make the remarks of the previous paragraph precise, let us introduce new coordinates $\tilde{x}_{\mu(i, j)}, \tilde{x}_{v(j, k)}$, and $\tilde{x}_{\zeta(i, k)}$ that are related to the old coordinates by 


$$
\begin{aligned}
\tilde{x}_{\mu(i, j)} & =x_{\mu(i, j)}, \\
\tilde{x}_{v(j, k)} & =x_{v(j, k)}, \\
\tilde{x}_{\zeta(i, k)} & =x_{\zeta(i, k)}-\frac{1}{2} \varphi_{\zeta(i, k)} .
\end{aligned}
$$

We use a tilde to denote other objects associated with this coordinate system so that, for example, $\tilde{\partial}_{\mu(i, j)}$ denotes the partial derivative with respect to $\tilde{x}_{\mu(i, j)}$. With this convention, we may express the operator $\Omega_{\zeta(i, k)}^{(z)}$ in terms of the new coordinate system as follows.

Proposition 4.1. We have

$$
\begin{aligned}
\Omega_{\zeta(i, k)}^{(z)}= & \tilde{\Delta}_{\zeta(i, k)}-z \tilde{\partial}_{\zeta(i, k)}+\frac{1}{2} \sum_{p=1}^{a} \tilde{P}_{i, p} \tilde{\partial}_{\zeta(p, k)}-\frac{1}{2} \sum_{r=1}^{c} \tilde{Q}_{r, k} \tilde{\partial}_{\zeta(i, r)} \\
& -\frac{1}{4} \sum_{p=1}^{a} \sum_{r=1}^{c} \tilde{\varphi}_{\zeta(p, r)} \tilde{\partial}_{\zeta(i, r)} \tilde{\partial}_{\zeta(p, k)} .
\end{aligned}
$$

Proof. It follows from the chain rule that we have

$$
\begin{aligned}
& \partial_{\mu(i, j)}=\tilde{\partial}_{\mu(i, j)}-\frac{1}{2} \sum_{r=1}^{c} \tilde{x}_{v(j, r)} \tilde{\partial}_{\zeta(i, r)}, \\
& \partial_{v(j, k)}=\tilde{\partial}_{v(j, k)}-\frac{1}{2} \sum_{p=1}^{a} \tilde{x}_{\mu(p, j)} \tilde{\partial}_{\zeta(p, k)}, \\
& \partial_{\zeta(i, k)}=\partial_{\zeta(i, k)} .
\end{aligned}
$$

Thus

$$
\begin{aligned}
\partial_{\mu(i, j)} \partial_{v(j, k)}= & \tilde{\partial}_{\mu(i, j)} \tilde{\partial}_{v(j, k)}-\frac{1}{2} \sum_{p=1}^{a} \tilde{\partial}_{\mu(i, j)} \tilde{x}_{\mu(p, j)} \tilde{\partial}_{\zeta(p, k)}-\frac{1}{2} \sum_{r=1}^{c} \tilde{x}_{v(j, r)} \tilde{\partial}_{v(j, k)} \tilde{\partial}_{\zeta(i, r)} \\
& +\frac{1}{4} \sum_{p=1}^{a} \sum_{r=1}^{c} \tilde{x}_{\mu(p, j)} \tilde{x}_{v(j, r)} \tilde{\partial}_{\zeta(i, r)} \tilde{\partial}_{\zeta(p, k)} \\
= & \tilde{\partial}_{\mu(i, j)} \tilde{\partial}_{v(j, k)}-\frac{1}{2} \tilde{\partial}_{\zeta(i, k)}-\frac{1}{2} \sum_{p=1}^{a} \tilde{x}_{\mu(p, j)} \tilde{\partial}_{\mu(i, j)} \tilde{\partial}_{\zeta(p, k)} \\
& -\frac{1}{2} \sum_{r=1}^{c} \tilde{x}_{v(j, r)} \tilde{\partial}_{v(j, k)} \tilde{\partial}_{\zeta(i, r)}+\frac{1}{4} \sum_{p=1}^{a} \sum_{r=1}^{c} \tilde{x}_{\mu(p, j)} \tilde{x}_{v(j, r)} \tilde{\partial}_{\zeta(i, r)} \tilde{\partial}_{\zeta(p, k)}
\end{aligned}
$$


and so, recalling that $z_{0}=b / 2$,

$$
\begin{aligned}
\Delta_{\zeta(i, k)}= & \tilde{\Delta}_{\zeta(i, k)}-z_{0} \tilde{\partial}_{\zeta(i, k)}-\frac{1}{2} \sum_{p=1}^{a} \tilde{P}_{i, p} \tilde{\partial}_{\zeta(p, k)}-\frac{1}{2} \sum_{r=1}^{c} \tilde{Q}_{r, k} \tilde{\partial}_{\zeta(i, r)} \\
& +\frac{1}{4} \sum_{p=1}^{a} \sum_{r=1}^{c} \tilde{\varphi}_{\zeta(p, r)} \tilde{\partial}_{\zeta(i, r)} \tilde{\partial}_{\zeta(p, k)} .
\end{aligned}
$$

Similarly,

$$
P_{i, p}=\tilde{P}_{i, p}-\frac{1}{2} \sum_{r=1}^{c} \tilde{\varphi}_{\zeta(p, r)} \tilde{\partial}_{\zeta(i, r)}
$$

and so

$$
\sum_{p=1}^{a} P_{i, p} \partial_{\zeta(p, k)}=\sum_{p=1}^{a} \tilde{P}_{i, p} \tilde{\partial}_{\zeta(p, k)}-\frac{1}{2} \sum_{p=1}^{a} \sum_{r=1}^{c} \tilde{\varphi}_{\zeta(p, r)} \tilde{\partial}_{\zeta(i, r)} \tilde{\partial}_{\zeta(p, k)} .
$$

The stated evaluation follows on combining these.

In order to compare the expression obtained in Proposition 4.1 with the standard expression for the Heisenberg Ultrahyperbolic operator, we take $a=$ $c=1$, write $t$ for $x_{\zeta(1,1)}, x_{j}$ for $x_{\mu(1, j)}$, and $y_{j}$ for $x_{v(j, 1)}$. We obtain

$$
\Omega^{(z)}=\Delta+\frac{1}{2}\left(\mathbf{E}_{x}-\mathbf{E}_{y}\right) \frac{\partial}{\partial t}-\frac{1}{4} \varphi \frac{\partial^{2}}{\partial t^{2}}-z \frac{\partial}{\partial t},
$$

where $\mathbf{E}_{x}$ and $\mathbf{E}_{y}$ are the Euler operators for the indicated sets of variables. The standard expression for the Heisenberg Laplacian results from this by a change of real form, taking $x_{j}$ and $y_{j}$ to be complex conjugates of one another and replacing $t$ by $\sqrt{-1} t$.

\section{Conjugate Systems}

To a number $l \geq 2$ and a triple $(a, b, c)$ such that $a, b, c \geq 1$ and $a+b+c=$ $l+1$ we have associated an $\Omega$-system consisting of $a c$ differential operators depending on a parameter $z$. The differential operators in this system live on the group $N$ consisting of matrices of the form

$$
x=\left(\begin{array}{ccc}
I_{a} & X & T \\
0 & I_{b} & Y \\
0 & 0 & I_{c}
\end{array}\right)
$$


The group $N$ is a subgroup of $\mathrm{SL}(l+1)$ and this latter group has an automorphism $g \mapsto \tilde{g}$ given by $\tilde{g}=J\left(g^{-1}\right)^{\top} J^{-1}$, where $\top$ denotes the transpose and

$$
J=\left(\begin{array}{rrr}
0 & 0 & I_{c} \\
0 & -I_{b} & 0 \\
I_{a} & 0 & 0
\end{array}\right)
$$

One computes that

$$
\tilde{x}=\left(\begin{array}{ccc}
I_{c} & Y^{\top} & (X Y-T)^{\top} \\
0 & I_{b} & X^{\top} \\
0 & 0 & I_{a}
\end{array}\right)
$$

and so the image of $N$ under this automorphism is the subgroup $\tilde{N}$ associated to the triple $(c, b, a)$. It is natural to expect that there is a relationship between the $\Omega$-systems associated to $N$ and to $\tilde{N}$, and this is indeed true. That it must be so may be derived from the general theory of conformally invariant systems, but in this section we instead establish it directly. With the convention that a tilde denotes objects associated with $\tilde{N}$, the relation between the coordinates on the two groups is

$$
\begin{aligned}
& \tilde{x}_{\mu(k, j)}=x_{v(j, k)}, \\
& \tilde{x}_{v(j, i)}=x_{\mu(i, j)}, \\
& \tilde{x}_{\zeta(k, i)}=\varphi_{\zeta(i, k)}-x_{\zeta(i, k)} .
\end{aligned}
$$

Proposition 5.1. We have $\tilde{\mathbf{\Omega}}_{\zeta(k, i)}^{(z)}=\Omega_{\zeta(i, k)}^{(-z)}$.

Proof. The relation between the coordinates $x$ and $\tilde{x}$ given above imply that

$$
\begin{aligned}
& \partial_{\mu(i, j)}=\tilde{\partial}_{v(j, i)}+\sum_{r=1}^{c} \tilde{x}_{\mu(r, j)} \tilde{\partial}_{\zeta(r, i)}, \\
& \partial_{v(j, k)}=\tilde{\partial}_{\mu(k, j)}+\sum_{p=1}^{a} \tilde{x}_{v(j, p)} \tilde{\partial}_{\zeta(k, p)}, \\
& \partial_{\zeta(i, k)}=-\tilde{\partial}_{\zeta(k, i)} .
\end{aligned}
$$


Thus

$$
\begin{aligned}
\Delta_{\zeta(i, k)}= & \tilde{\Delta}_{\zeta(k, i)}+\sum_{p=1}^{a} \sum_{j=1}^{b} \tilde{\partial}_{v(j, i)} \tilde{x}_{v(j, p)} \tilde{\partial}_{\zeta(k, p)}+\sum_{j=1}^{b} \sum_{r=1}^{c} \tilde{x}_{\mu(r, j)} \tilde{\partial}_{\mu(k, j)} \tilde{\partial}_{\zeta(r, i)} \\
& +\sum_{p=1}^{a} \sum_{r=1}^{c} \tilde{\varphi}_{\zeta(r, p)} \tilde{\partial}_{\zeta(r, i)} \tilde{\partial}_{\zeta(k, p)} \\
= & \tilde{\Delta}_{\zeta(k, i)}+\sum_{p=1}^{a} \sum_{j=1}^{b}\left(\delta_{i p}+\tilde{x}_{v(j, p)} \tilde{\partial}_{v(j, i)}\right) \tilde{\partial}_{\zeta(k, p)}+\sum_{r=1}^{c} \tilde{P}_{k, r} \tilde{\partial}_{\zeta(r, i)} \\
& +\sum_{p=1}^{a} \sum_{r=1}^{c} \tilde{\varphi}_{\zeta(r, p)} \tilde{\partial}_{\zeta(r, i)} \tilde{\partial}_{\zeta(k, p)} \\
= & \tilde{\Delta}_{\zeta(k, i)}+b \tilde{\partial}_{\zeta(k, i)}+\sum_{p=1}^{a} \sum_{j=1}^{b} \tilde{x}_{v(j, p)} \tilde{\partial}_{v(j, i)} \tilde{\partial}_{\zeta(k, p)}+\sum_{r=1}^{c} \tilde{P}_{k, r} \tilde{\partial}_{\zeta(r, i)} \\
& +\sum_{p=1}^{a} \sum_{r=1}^{c} \tilde{\varphi}_{\zeta(r, p)} \tilde{\partial}_{\zeta(r, i)} \tilde{\partial}_{\zeta(k, p)} .
\end{aligned}
$$

Now

$$
\begin{aligned}
P_{i, p} & =\sum_{j=1}^{b} x_{\mu(p, j)} \partial_{\mu(i, j)} \\
& =\sum_{j=1}^{b} \tilde{x}_{v(j, p)}\left(\tilde{\partial}_{v(j, i)}+\sum_{r=1}^{c} \tilde{x}_{\mu(r, j)} \tilde{\partial}_{\zeta(r, i)}\right) \\
& =\sum_{j=1}^{b} \tilde{x}_{v(j, p)} \tilde{\partial}_{v(j, i)}+\sum_{r=1}^{c} \tilde{\varphi}_{\zeta(r, p)} \tilde{\partial}_{\zeta(r, i)}
\end{aligned}
$$

and so

$$
\sum_{p=1}^{a} P_{i, p} \partial_{\zeta(p, k)}=-\sum_{p=1}^{a} \sum_{j=1}^{b} \tilde{x}_{v(j, p)} \tilde{\partial}_{v(j, i)} \tilde{\partial}_{\zeta(k, p)}-\sum_{p=1}^{a} \sum_{r=1}^{c} \tilde{\varphi}_{\zeta(r, p)} \tilde{\partial}_{\zeta(r, i)} \tilde{\partial}_{\zeta(k, p)} .
$$

By adding the above evaluations, we obtain

$$
\Delta_{\zeta(i, k)}+\sum_{p=1}^{a} P_{i, p} \partial_{\zeta(p, k)}=\tilde{\Delta}_{\zeta(k, i)}+b \tilde{\partial}_{\zeta(k, i)}+\sum_{r=1}^{c} \tilde{P}_{k, r} \tilde{\partial}_{\zeta(r, i)}
$$


Finally, recalling that $z_{0}=b / 2$, we have

$$
\begin{aligned}
\Omega_{\zeta(i, k)}^{(z)} & =\tilde{\Delta}_{\zeta(k, i)}+b \tilde{\partial}_{\zeta(k, i)}+\left(z-z_{0}\right) \tilde{\partial}_{\zeta(k, i)}+\sum_{r=1}^{c} \tilde{P}_{k, r} \tilde{\partial}_{\zeta(r, i)} \\
& =\tilde{\Delta}_{\zeta(k, i)}+\left(z+z_{0}\right) \tilde{\partial}_{\zeta(k, i)}+\sum_{r=1}^{c} \tilde{P}_{k, r} \tilde{\partial}_{\zeta(r, i)} \\
& =\tilde{\Delta}_{\zeta(k, i)}-\left(-z-z_{0}\right) \tilde{\partial}_{\zeta(k, i)}+\sum_{r=1}^{c} \tilde{P}_{k, r} \tilde{\partial}_{\zeta(r, i)} \\
& =\tilde{\boldsymbol{\Omega}}_{\zeta(k, i)}^{(-z)},
\end{aligned}
$$

as required.

We refer to the $\Omega$-systems associated with the groups $N$ and $\tilde{N}$ with the parameters $z$ and $-z$, respectively, as conjugate $\Omega$-systems. Proposition 5.1 implies that these systems are identical, up to a change of coordinates, and thus that a solution to one easily yields a solution to the other.

\section{Integrability of the $\Omega$-System}

The major goal in this section is to show that the $\Omega$-system in evolutionary form is free of integrability conditions. As with any system having this property, we then obtain a general form for the solution with given initial conditions.

We assume in this section that $z-z_{0} \notin \sigma(F)$ so that the operator $\left(z-z_{0}\right) I-F$ on $\mathbf{C}\left[x_{\mu}\right] \otimes \mathbf{C}^{R_{13}}$ is invertible. For brevity, let $w=z-z_{0}$. Recall that $w I-F$ is a block sum of operators of the form $w I-P$ acting on $\mathbf{C}\left[x_{\mu}\right] \otimes \mathbf{C}^{a}$. Each of these operators is also invertible and so we have an operator

$$
(w I-P)^{-1}: \mathbf{C}\left[x_{\mu}\right] \otimes \mathbf{C}^{a} \rightarrow \mathbf{C}\left[x_{\mu}\right] \otimes \mathbf{C}^{a} .
$$

Note that the action of $(w I-P)$ on $\mathbf{C}\left[x_{\mu}\right] \otimes \mathbf{C}^{a}$ is locally finite and it follows that the same is true for $(w I-P)^{-1}$. Since $\mathbf{C}\left[x_{\mu}\right] \otimes \mathbf{C}^{a}$ may be thought of as the space of $a$-tuples of elements of $\mathbf{C}\left[x_{\mu}\right],(w I-P)^{-1}$ may be identified with an $a$-by- $a$ matrix of operators on $\mathbf{C}\left[x_{\mu}\right]$ in the usual way. We write the $(i, p)$-entry of this matrix as $(w I-P)_{i, p}^{\dagger}$. Thus $(w I-P)_{i, p}^{\dagger}$ is an operator from $\mathbf{C}\left[x_{\mu}\right]$ to itself for each $i$ and $p$, and we have

$$
\sum_{s=1}^{a}(w I-P)_{i, s}(w I-P)_{s, p}^{\dagger}=\delta_{i p} I
$$


and

$$
\sum_{s=1}^{a}(w I-P)_{i, s}^{\dagger}(w I-P)_{s, p}=\delta_{i p} I .
$$

For $1 \leq i \leq a$ and $1 \leq k \leq c$, we define

$$
V_{\zeta(i, k)}=\sum_{s=1}^{a}(w I-P)_{i, s}^{\dagger} \Delta_{\zeta(s, k)} .
$$

Each $V_{\zeta(i, k)}$ is an operator from $\mathbf{C}\left[x_{\mu}, x_{v}\right]$ to itself. In this expression, we have extended $(w I-P)_{i, p}^{\dagger}$ to the operator $(w I-P)_{i, p}^{\dagger} \otimes I$ on $\mathbf{C}\left[x_{\mu}, x_{v}\right] \cong \mathbf{C}\left[x_{\mu}\right] \otimes \mathbf{C}\left[x_{v}\right]$. We shall continue to make such extensions silently in what follows.

Lemma 6.1. The evolutionary form of the $\Omega$-system is

$$
\frac{\partial \Phi}{\partial x_{\zeta}}=V_{\zeta} \bullet \Phi
$$

for all $\zeta \in R_{13}$.

Proof. In Section 2, we wrote the $\Omega$-system in vector form as

$$
\left[\Delta_{\zeta} \bullet \Phi\right]=(w I-F)\left[\partial_{\zeta} \bullet \Phi\right]
$$

and observed that

$$
F_{\zeta(i, k), \zeta(p, r)}=\delta_{k r} P_{i, p}
$$

This is equivalent to

$$
\begin{aligned}
\Delta_{\zeta(i, k)} \bullet \Phi & =\sum_{p=1}^{a} \sum_{r=1}^{c}(w I-F)_{\zeta(i, k), \zeta(p, r)} \partial_{\zeta(p, r)} \bullet \Phi \\
& =\sum_{p=1}^{a} \sum_{r=1}^{c} \delta_{k r}(w I-P)_{i, p} \partial_{\zeta(p, r)} \bullet \Phi \\
& =\sum_{p=1}^{a}(w I-P)_{i, p} \partial_{\zeta(p, k)} \bullet \Phi .
\end{aligned}
$$

We multiply by $(w I-P)_{m, i}^{\dagger}$ on the left and sum over $i$ to obtain

$$
V_{\zeta(m, k)} \bullet \Phi=\partial_{\zeta(m, k)} \bullet \Phi
$$

as required. 
It follows from this observation that integrability conditions for the $\Omega$-system would arise from non-zero commutators $\left[V_{\zeta}, V_{\xi}\right]$ if there were any. Thus we next investigate these commutators.

LeMma 6.2. We have

$$
\left[V_{\zeta(i, k)}, P_{m, p}\right]=\delta_{i p} V_{\zeta(m, k)}
$$

for all $1 \leq i, m, p \leq a$ and $1 \leq k \leq c$.

Proof. We have

$$
\begin{aligned}
\sum_{i_{2}=1}^{a} & (w I-P)_{i_{1}, i_{2}}\left[V_{\zeta\left(i_{2}, k\right)}, P_{i_{3}, i_{4}}\right] \\
& =\sum_{i_{2}=1}^{a}(w I-P)_{i_{1}, i_{2}} V_{\zeta\left(i_{2}, k\right)} P_{i_{3}, i_{4}}-\sum_{i_{2}=1}^{a}(w I-P)_{i_{1}, i_{2}} P_{i_{3}, i_{4}} V_{\zeta\left(i_{2}, k\right)} \\
& =\Delta_{\zeta\left(i_{1}, k\right)} P_{i_{3}, i_{4}}-\sum_{i_{2}=1}^{a} P_{i_{3}, i_{4}}(w I-P)_{i_{1}, i_{2}} V_{\zeta\left(i_{2}, k\right)}-\sum_{i_{2}=1}^{a}\left[(w I-P)_{i_{1}, i_{2}}, P_{i_{3}, i_{4}}\right] V_{\zeta\left(i_{2}, k\right)} \\
& =\Delta_{\zeta\left(i_{1}, k\right)} P_{i_{3}, i_{4}}-P_{i_{3}, i_{4}} \Delta_{\zeta\left(i_{1}, k\right)}+\sum_{i_{2}=1}^{a}\left[P_{i_{1}, i_{2}}, P_{i_{3}, i_{4}}\right] V_{\zeta\left(i_{2}, k\right)} \\
& =\left[\Delta_{\zeta\left(i_{1}, k\right)}, P_{i_{3}, i_{4}}\right]+\sum_{i_{2}=1}^{a}\left(\delta_{i_{1} i_{4}} P_{i_{3}, i_{2}}-\delta_{i_{2} i_{3}} P_{i_{1}, i_{4}}\right) V_{\zeta\left(i_{2}, k\right)} \\
& =\delta_{i_{1} i_{4}} \Delta_{\zeta\left(i_{3}, k\right)}-P_{i_{1}, i_{4}} V_{\zeta\left(i_{3}, k\right)}+\delta_{i_{1} i_{4}} \sum_{i_{2}=1}^{a} P_{i_{3}, i_{2}} V_{\zeta\left(i_{2}, k\right)} \\
& =\delta_{i_{1} i_{4}} \Delta_{\zeta\left(i_{3}, k\right)}-P_{i_{1}, i_{4}} V_{\zeta\left(i_{3}, k\right)}+\delta_{i_{1} i_{4}} \sum_{i_{2}=1}^{a}(P-w I)_{i_{3}, i_{2}} V_{\zeta\left(i_{2}, k\right)}+w \delta_{i_{1} i_{4}} \sum_{i_{2}=1}^{a} \delta_{i_{3} i_{2}} V_{\zeta\left(i_{2}, k\right)} \\
= & \delta_{i_{1} i_{4}} \Delta_{\zeta\left(i_{3}, k\right)}-P_{i_{1}, i_{4}} V_{\zeta\left(i_{3}, k\right)}-\delta_{i_{1} i_{4}} \Delta_{\zeta\left(i_{3}, k\right)}+w \delta_{i_{1} i_{4}} V_{\zeta\left(i_{3}, k\right)} \\
= & (w I-P)_{i_{1}, i_{4}} V_{\zeta\left(i_{3}, k\right)} .
\end{aligned}
$$

Note that we have used Lemma 3.1 to evaluate $\left[P_{i_{1}, i_{2}}, P_{i_{3}, i_{4}}\right]$ and Lemma 3.2 to evaluate $\left[\Delta_{\zeta\left(i_{1}, k\right)}, P_{i_{3}, i_{4}}\right]$ during this computation. We now multiply on the left by $(w I-P)_{i_{5}, i_{1}}^{\dagger}$ and sum over $i_{1}$ to obtain

$$
\left[V_{\zeta\left(i_{5}, k\right)}, P_{i_{3}, i_{4}}\right]=\delta_{i_{5} i_{4}} V_{\zeta\left(i_{3}, k\right)} .
$$

This is the stated identity but for replacement of the subscripts. 
LEMMA 6.3. We have

$$
\left[\Delta_{\zeta(i, k)}, V_{\zeta(p, r)}\right]=V_{\zeta(p, k)} V_{\zeta(i, r)}
$$

for all $1 \leq i, p \leq a$ and $1 \leq k, r \leq c$.

Proof. We have

$$
\begin{aligned}
\sum_{i_{2}=1}^{a}(w I-P)_{i_{1}, i_{2}}\left[V_{\zeta\left(i_{2}, k_{1}\right)}, \Delta_{\zeta\left(i_{3}, k_{2}\right)}\right] & \\
= & \sum_{i_{2}=1}^{a}(w I-P)_{i_{1}, i_{2}} V_{\zeta\left(i_{2}, k_{1}\right)} \Delta_{\zeta\left(i_{3}, k_{2}\right)}-\sum_{i_{2}=1}^{a}(w I-P)_{i_{1}, i_{2}} \Delta_{\zeta\left(i_{3}, k_{2}\right)} V_{\zeta\left(i_{2}, k_{1}\right)} \\
= & \Delta_{\zeta\left(i_{1}, k_{1}\right)} \Delta_{\zeta\left(i_{3}, k_{2}\right)}-\sum_{i_{2}=1}^{a} \Delta_{\zeta\left(i_{3}, k_{2}\right)}(w I-P)_{i_{1}, i_{2}} V_{\zeta\left(i_{2}, k_{1}\right)} \\
& -\sum_{i_{2}=1}^{a}\left[(w I-P)_{i_{1}, i_{2}}, \Delta_{\zeta\left(i_{3}, k_{2}\right)}\right] V_{\zeta\left(i_{2}, k_{1}\right)} \\
= & \Delta_{\zeta\left(i_{1}, k_{1}\right)} \Delta_{\zeta\left(i_{3}, k_{2}\right)}-\Delta_{\zeta\left(i_{3}, k_{2}\right)} \Delta_{\zeta\left(i_{1}, k_{1}\right)}+\sum_{i_{2}=1}^{a}\left[P_{\left.i_{1}, i_{2}, \Delta_{\zeta\left(i_{3}, k_{2}\right)}\right] V_{\zeta\left(i_{2}, k_{1}\right)}}\right. \\
= & -\sum_{i_{2}=1}^{a} \delta_{i_{2} i_{3}} \Delta_{\zeta\left(i_{1}, k_{2}\right)} V_{\zeta\left(i_{2}, k_{1}\right)} \\
= & -\Delta_{\zeta\left(i_{1}, k_{2}\right)} V_{\zeta\left(i_{3}, k_{1}\right)} .
\end{aligned}
$$

We have used Lemma 3.2 to evaluate $\left[P_{i_{1}, i_{2}}, \Delta_{\zeta\left(i_{3}, k_{2}\right)}\right]$ during this computation.

We now multiply on the left by $(w I-P)_{i_{4}, i_{1}}^{\dagger}$ and sum over $i_{1}$ to obtain

$$
\left[V_{\zeta\left(i_{4}, k_{1}\right)}, \Delta_{\zeta\left(i_{3}, k_{2}\right)}\right]=-V_{\zeta\left(i_{4}, k_{2}\right)} V_{\zeta\left(i_{3}, k_{1}\right)}
$$

or equivalently

$$
\left[\Delta_{\zeta\left(i_{3}, k_{2}\right)}, V_{\zeta\left(i_{4}, k_{1}\right)}\right]=V_{\zeta\left(i_{4}, k_{2}\right)} V_{\zeta\left(i_{3}, k_{1}\right)} .
$$

This is the stated identity but for replacement of the subscripts.

THEOREM 6.4. We have

$$
\left[V_{\zeta(i, k)}, V_{\zeta(p, r)}\right]=0
$$

for all $1 \leq i, p \leq a$ and $1 \leq k, r \leq c$. 
Proof. We have

$$
\begin{aligned}
& \sum_{i_{2}, i_{4}=1}^{a}(w I-P)_{i_{1}, i_{2}}(w I-P)_{i_{3}, i_{4}}\left[V_{\zeta\left(i_{4}, k_{1}\right)}, V_{\zeta\left(i_{2}, k_{2}\right)}\right] \\
& =\sum_{i_{2}, i_{4}=1}^{a}(w I-P)_{i_{1}, i_{2}}(w I-P)_{i_{3}, i_{4}} V_{\zeta\left(i_{4}, k_{1}\right)} V_{\zeta\left(i_{2}, k_{2}\right)} \\
& -\sum_{i_{2}, i_{4}=1}^{a}(w I-P)_{i_{1}, i_{2}}(w I-P)_{i_{3}, i_{4}} V_{\zeta\left(i_{2}, k_{2}\right)} V_{\zeta\left(i_{4}, k_{1}\right)} \\
& =\sum_{i_{2}=1}^{a}(w I-P)_{i_{1}, i_{2}} \Delta_{\zeta\left(i_{3}, k_{1}\right)} V_{\zeta\left(i_{2}, k_{2}\right)} \\
& -\sum_{i_{2}, i_{4}=1}^{a}(w I-P)_{i_{3}, i_{4}}(w I-P)_{i_{1}, i_{2}} V_{\zeta\left(i_{2}, k_{2}\right)} V_{\zeta\left(i_{4}, k_{1}\right)} \\
& -\sum_{i_{2}, i_{4}=1}^{a}\left[(w I-P)_{i_{1}, i_{2}},(w I-P)_{i_{3}, i_{4}}\right] V_{\zeta\left(i_{2}, k_{2}\right)} V_{\zeta\left(i_{4}, k_{1}\right)} \\
& =\sum_{i_{2}=1}^{a}(w I-P)_{i_{1}, i_{2}} \Delta_{\zeta\left(i_{3}, k_{1}\right)} V_{\zeta\left(i_{2}, k_{2}\right)}-\sum_{i_{4}=1}^{a}(w I-P)_{i_{3}, i_{4}} \Delta_{\zeta\left(i_{1}, k_{2}\right)} V_{\zeta\left(i_{4}, k_{1}\right)} \\
& -\sum_{i_{2}, i_{4}=1}^{a}\left[P_{i_{1}, i_{2}}, P_{i_{3}, i_{4}}\right] V_{\zeta\left(i_{2}, k_{2}\right)} V_{\zeta\left(i_{4}, k_{1}\right)} \\
& =\sum_{i_{2}=1}^{a}(w I-P)_{i_{1}, i_{2}} \Delta_{\zeta\left(i_{3}, k_{1}\right)} V_{\zeta\left(i_{2}, k_{2}\right)}-\sum_{i_{4}=1}^{a}(w I-P)_{i_{3}, i_{4}} \Delta_{\zeta\left(i_{1}, k_{2}\right)} V_{\zeta\left(i_{4}, k_{1}\right)} \\
& -\sum_{i_{2}, i_{4}=1}^{a}\left(\delta_{i_{1} i_{4}} P_{i_{3}, i_{2}}-\delta_{i_{2} i_{3}} P_{i_{1}, i_{4}}\right) V_{\zeta\left(i_{2}, k_{2}\right)} V_{\zeta\left(i_{4}, k_{1}\right)} \\
& =\sum_{i_{2}=1}^{a}(w I-P)_{i_{1}, i_{2}} \Delta_{\zeta\left(i_{3}, k_{1}\right)} V_{\zeta\left(i_{2}, k_{2}\right)}-\sum_{i_{4}=1}^{a}(w I-P)_{i_{3}, i_{4}} \Delta_{\zeta\left(i_{1}, k_{2}\right)} V_{\zeta\left(i_{4}, k_{1}\right)} \\
& -\sum_{i_{2}=1}^{a} P_{i_{3}, i_{2}} V_{\zeta\left(i_{2}, k_{2}\right)} V_{\zeta\left(i_{1}, k_{1}\right)}+\sum_{i_{4}=1}^{a} P_{i_{1}, i_{4}} V_{\zeta\left(i_{3}, k_{2}\right)} V_{\zeta\left(i_{4}, k_{1}\right)} \\
& =1-2-3+4 \text {. }
\end{aligned}
$$


In this computation we have used Lemma 3.1 to evaluate $\left[P_{i_{1}, i_{2}}, P_{i_{3}, i_{4}}\right]$. The four boxed terms on the last line stand for the four terms in the previous line in the obvious way.

The next step is to further simplify each of the four terms in the expression that we have just found. We have

$$
\begin{aligned}
1 & =\sum_{i_{2}=1}^{a}(w I-P)_{i_{1}, i_{2}} \Delta_{\zeta\left(i_{3}, k_{1}\right)} V_{\zeta\left(i_{2}, k_{2}\right)} \\
& =\sum_{i_{2}=1}^{a} \Delta_{\zeta\left(i_{3}, k_{1}\right)}(w I-P)_{i_{1}, i_{2}} V_{\zeta\left(i_{2}, k_{2}\right)}+\sum_{i_{2}=1}^{a}\left[(w I-P)_{i_{1}, i_{2}}, \Delta_{\zeta\left(i_{3}, k_{1}\right)}\right] V_{\zeta\left(i_{2}, k_{2}\right)} \\
& =\Delta_{\zeta\left(i_{3}, k_{1}\right)} \Delta_{\zeta\left(i_{1}, k_{2}\right)}-\sum_{i_{2}=1}^{a}\left[P_{i_{1}, i_{2}}, \Delta_{\zeta\left(i_{3}, k_{1}\right)}\right] V_{\zeta\left(i_{2}, k_{2}\right)} \\
& =\Delta_{\zeta\left(i_{3}, k_{1}\right)} \Delta_{\zeta\left(i_{1}, k_{2}\right)}+\sum_{i_{2}=1}^{a} \delta_{i_{2} i_{3}} \Delta_{\zeta\left(i_{1}, k_{1}\right)} V_{\zeta\left(i_{2}, k_{2}\right)} \\
& =\Delta_{\zeta\left(i_{3}, k_{1}\right)} \Delta_{\zeta\left(i_{1}, k_{2}\right)}+\Delta_{\zeta\left(i_{1}, k_{1}\right)} V_{\zeta\left(i_{3}, k_{2}\right)}
\end{aligned}
$$

We have used Lemma 3.2 to evaluate $\left[P_{i_{1}, i_{2}}, \Delta_{\zeta\left(i_{3}, k_{1}\right)}\right]$ in this computation. Next we have

$$
\begin{aligned}
2 & =\sum_{i_{4}=1}^{a}(w I-P)_{i_{3}, i_{4}} \Delta_{\zeta\left(i_{1}, k_{2}\right)} V_{\zeta\left(i_{4}, k_{1}\right)} \\
& =\sum_{i_{4}=1}^{a} \Delta_{\zeta\left(i_{1}, k_{2}\right)}(w I-P)_{i_{3}, i_{4}} V_{\zeta\left(i_{4}, k_{1}\right)}+\sum_{i_{4}=1}^{a}\left[(w I-P)_{i_{3}, i_{4}}, \Delta_{\zeta\left(i_{1}, k_{2}\right)}\right] V_{\zeta\left(i_{4}, k_{1}\right)} \\
& =\Delta_{\zeta\left(i_{1}, k_{2}\right)} \Delta_{\zeta\left(i_{3}, k_{1}\right)}-\sum_{i_{4}=1}^{a}\left[P_{i_{3}, i_{4}}, \Delta_{\zeta\left(i_{1}, k_{2}\right)}\right] V_{\zeta\left(i_{4}, k_{1}\right)} \\
& =\Delta_{\zeta\left(i_{1}, k_{2}\right)} \Delta_{\zeta\left(i_{3}, k_{1}\right)}+\sum_{i_{4}=1}^{a} \delta_{i_{1} i_{4}} \Delta_{\zeta\left(i_{3}, k_{2}\right)} V_{\zeta\left(i_{4}, k_{1}\right)} \\
& =\Delta_{\zeta\left(i_{1}, k_{2}\right)} \Delta_{\zeta\left(i_{3}, k_{1}\right)}+\Delta_{\zeta\left(i_{3}, k_{2}\right)} V_{\zeta\left(i_{1}, k_{1}\right)}
\end{aligned}
$$

We have used Lemma 3.2 to evaluate $\left[P_{i_{3}, i_{4}}, \Delta_{\zeta\left(i_{1}, k_{2}\right)}\right]$ in this computation. The third term is 


$$
\begin{aligned}
3 & =\sum_{i_{2}=1}^{a} P_{i_{3}, i_{2}} V_{\zeta\left(i_{2}, k_{2}\right)} V_{\zeta\left(i_{1}, k_{1}\right)} \\
& =\sum_{i_{2}=1}^{a}(P-w I)_{i_{3}, i_{2}} V_{\zeta\left(i_{2}, k_{2}\right)} V_{\zeta\left(i_{1}, k_{1}\right)}+w \sum_{i_{2}=1}^{a} \delta_{i_{3} i_{2}} V_{\zeta\left(i_{2}, k_{2}\right)} V_{\zeta\left(i_{1}, k_{1}\right)} \\
& =-\Delta_{\zeta\left(i_{3}, k_{2}\right)} V_{\zeta\left(i_{1}, k_{1}\right)}+w V_{\zeta\left(i_{3}, k_{2}\right)} V_{\zeta\left(i_{1}, k_{1}\right)}
\end{aligned}
$$

Finally, the fourth term is

$$
\begin{aligned}
4 & =\sum_{i_{4}=1}^{a} P_{i_{1}, i_{4}} V_{\zeta\left(i_{3}, k_{2}\right)} V_{\zeta\left(i_{4}, k_{1}\right)} \\
= & \sum_{i_{4}=1}^{a} V_{\zeta\left(i_{3}, k_{2}\right)} P_{i_{1}, i_{4}} V_{\zeta\left(i_{4}, k_{1}\right)}+\sum_{i_{4}=1}^{a}\left[P_{i_{1}, i_{4}}, V_{\zeta\left(i_{3}, k_{2}\right)}\right] V_{\zeta\left(i_{4}, k_{1}\right)} \\
= & \sum_{i_{4}=1}^{a} V_{\zeta\left(i_{3}, k_{2}\right)}(P-w I)_{i_{1}, i_{4}} V_{\zeta\left(i_{4}, k_{1}\right)} \\
& +w \sum_{i_{4}=1}^{a} \delta_{i_{1} i_{4}} V_{\zeta\left(i_{3}, k_{2}\right)} V_{\zeta\left(i_{4}, k_{1}\right)}-\sum_{i_{4}=1}^{a} \delta_{i_{3} i_{4}} V_{\zeta\left(i_{1}, k_{2}\right)} V_{\zeta\left(i_{4}, k_{1}\right)} \\
= & -V_{\zeta\left(i_{3}, k_{2}\right)} \Delta_{\zeta\left(i_{1}, k_{1}\right)}+w V_{\zeta\left(i_{3}, k_{2}\right)} V_{\zeta\left(i_{1}, k_{1}\right)}-V_{\zeta\left(i_{1}, k_{2}\right)} V_{\zeta\left(i_{3}, k_{1}\right)} .
\end{aligned}
$$

In this computation, we have used Lemma 6.2 to evaluate $\left[P_{i_{1}, i_{4}}, V_{\zeta\left(i_{3}, k_{2}\right)}\right]$.

The next step is to recombine the four term that have just been simplified. The result is that

$$
\begin{aligned}
\sum_{i_{2}, i_{4}=1}^{a}( & w I-P)_{i_{1}, i_{2}}(w I-P)_{i_{3}, i_{4}}\left[V_{\zeta\left(i_{4}, k_{1}\right)}, V_{\zeta\left(i_{2}, k_{2}\right)}\right] \\
= & \left(\Delta_{\zeta\left(i_{3}, k_{1}\right)} \Delta_{\zeta\left(i_{1}, k_{2}\right)}+\Delta_{\zeta\left(i_{1}, k_{1}\right)} V_{\zeta\left(i_{3}, k_{2}\right)}\right)-\left(\Delta_{\zeta\left(i_{1}, k_{2}\right)} \Delta_{\zeta\left(i_{3}, k_{1}\right)}+\Delta_{\zeta\left(i_{3}, k_{2}\right)} V_{\zeta\left(i_{1}, k_{1}\right)}\right) \\
& -\left(-\Delta_{\zeta\left(i_{3}, k_{2}\right)} V_{\zeta\left(i_{1}, k_{1}\right)}+w V_{\zeta\left(i_{3}, k_{2}\right)} V_{\zeta\left(i_{1}, k_{1}\right)}\right) \\
& +\left(-V_{\zeta\left(i_{3}, k_{2}\right)} \Delta_{\zeta\left(i_{1}, k_{1}\right)}+w V_{\zeta\left(i_{3}, k_{2}\right)} V_{\zeta\left(i_{1}, k_{1}\right)}-V_{\zeta\left(i_{1}, k_{2}\right)} V_{\zeta\left(i_{3}, k_{1}\right)}\right) \\
= & {\left[\Delta_{\zeta\left(i_{1}, k_{1}\right)}, V_{\zeta\left(i_{3}, k_{2}\right)}\right]-V_{\zeta\left(i_{1}, k_{2}\right)} V_{\zeta\left(i_{3}, k_{1}\right)} } \\
= & V_{\zeta\left(i_{3}, k_{1}\right)} V_{\zeta\left(i_{1}, k_{2}\right)}-V_{\zeta\left(i_{1}, k_{2}\right)} V_{\zeta\left(i_{3}, k_{1}\right)} \\
= & {\left[V_{\zeta\left(i_{3}, k_{1}\right)}, V_{\zeta\left(i_{1}, k_{2}\right)}\right] . }
\end{aligned}
$$


In this computation we have used Lemma 6.3 to evaluate $\left[\Delta_{\zeta\left(i_{1}, k_{1}\right)}, V_{\zeta\left(i_{3}, k_{2}\right)}\right]$. For emphasis, we have just deduced that

$$
\sum_{i_{2}, i_{4}=1}^{a}(w I-P)_{i_{1}, i_{2}}(w I-P)_{i_{3}, i_{4}}\left[V_{\zeta\left(i_{4}, k_{1}\right)}, V_{\zeta\left(i_{2}, k_{2}\right)}\right]=\left[V_{\zeta\left(i_{3}, k_{1}\right)}, V_{\zeta\left(i_{1}, k_{2}\right)}\right]
$$

It remains to see that this identity implies that $\left[V_{\zeta\left(i_{3}, k_{1}\right)}, V_{\zeta\left(i_{1}, k_{2}\right)}\right]=0$ for all $1 \leq i_{1}, i_{3} \leq a$ and $1 \leq k_{1}, k_{2} \leq c$.

Suppose that there are some indices $i_{1}, i_{3}, k_{1}, k_{2}$ such that $\left[V_{\zeta\left(i_{3}, k_{1}\right)}, V_{\zeta\left(i_{1}, k_{2}\right)}\right]$ $\neq 0$. Then we may find a polynomial $\psi \in \mathbf{C}\left[x_{\mu}, x_{v}\right]$ such that $\left[V_{\zeta\left(i_{3}, k_{1}\right)}, V_{\zeta\left(i_{1}, k_{2}\right)}\right] \bullet \psi$ $\neq 0$ for some choice of indices. Let us fix such $k_{1}$ and $k_{2}$ and consider the collection of all $\left[V_{\zeta\left(i_{3}, k_{1}\right)}, V_{\zeta\left(i_{1}, k_{2}\right)}\right] \bullet \psi$ as $i_{1}$ and $i_{3}$ vary between 1 and $a$. At least one of these objects is non-zero. Now note that it follows from the definition of the $V_{\zeta}$ and the usual formula for the inverse of a matrix in terms of its classical adjoint that each $\left[V_{\zeta\left(i_{3}, k_{1}\right)}, V_{\zeta\left(i_{1}, k_{2}\right)}\right] \bullet \psi$ lies in $\mathbf{C}(w)\left[x_{\mu}, x_{v}\right]$, the space of polynomials in $x_{\mu}$ and $x_{v}$ with coefficients that are rational functions of $w$. If $f \in \mathbf{C}(w)\left[x_{\mu}, x_{v}\right]$ is non-zero then we may write

$$
f=w^{\mathrm{e}_{\infty}(f)} \sum_{k=0}^{\infty} c_{k} w^{-k}
$$

where $\mathrm{e}_{\infty}(f) \in \mathbf{Z}, c_{k} \in \mathbf{C}\left[x_{\mu}, x_{v}\right]$ for all $k \geq 0$, and $c_{0} \neq 0$. The exponent $\mathrm{e}_{\infty}(f)$ is uniquely determined and is chosen so that

$$
\lim _{w \rightarrow \infty} w^{-\mathrm{e}_{\infty}(f)} f=c_{0}
$$

exists and is non-zero. Alternatively, $\mathrm{e}_{\infty}(f)$ is the order of growth of $f$ at $\infty$ in the $w$-plane. Let us consider the finite list of integers

$$
\mathrm{e}_{\infty}\left(\left[V_{\zeta\left(i_{3}, k_{1}\right)}, V_{\zeta\left(i_{1}, k_{2}\right)}\right] \bullet \psi\right)
$$

where we restrict $i_{1}$ and $i_{3}$ to those values for which $\left[V_{\zeta\left(i_{3}, k_{1}\right)}, V_{\zeta\left(i_{1}, k_{2}\right)}\right] \bullet \psi \neq 0$. Note that we have

$$
\lim _{w \rightarrow \infty} w^{2}\left[V_{\zeta\left(i_{3}, k_{1}\right)}, V_{\zeta\left(i_{1}, k_{2}\right)}\right] \bullet \psi=\left[\Delta_{\zeta\left(i_{3}, k_{1}\right)}, \Delta_{\zeta\left(i_{1}, k_{2}\right)}\right] \bullet \psi=0
$$

and so we have

$$
\mathrm{e}_{\infty}\left(\left[V_{\zeta\left(i_{3}, k_{1}\right)}, V_{\zeta\left(i_{1}, k_{2}\right)}\right] \bullet \psi\right) \leq-3
$$


for all allowable $i_{1}$ and $i_{3}$. Among all

$$
\mathrm{e}_{\infty}\left(\left[V_{\zeta\left(i_{3}, k_{1}\right)}, V_{\zeta\left(i_{1}, k_{2}\right)}\right] \bullet \psi\right)
$$

on the list, let $e$ be the largest that occurs and fix a choice of $i$ and $p$ so that

$$
\mathrm{e}_{\infty}\left(\left[V_{\zeta\left(p, k_{1}\right)}, V_{\zeta\left(i, k_{2}\right)}\right] \bullet \psi\right)=e .
$$

Consider the identity

$$
\sum_{i_{2}, i_{4}=1}^{a}(w I-P)_{i, i_{2}}(w I-P)_{p, i_{4}}\left[V_{\zeta\left(i_{4}, k_{1}\right)}, V_{\zeta\left(i_{2}, k_{2}\right)}\right] \bullet \psi=\left[V_{\zeta\left(p, k_{1}\right)}, V_{\zeta\left(i, k_{2}\right)}\right] \bullet \psi
$$

that follows from what was previously established. On the right-hand side, the order of growth at $\infty$ is exactly $e$. On the left-hand side, we have a sum of terms each of which is of the form $(w I-P)_{i, i_{2}}(w I-P)_{p, i_{4}}\left[V_{\zeta\left(i_{4}, k_{1}\right)}, V_{\zeta\left(i_{2}, k_{2}\right)}\right] \bullet \psi$. The order of growth of each $\left[V_{\zeta\left(i_{4}, k_{1}\right)}, V_{\zeta\left(i_{2}, k_{2}\right)}\right] \bullet \psi$ at $\infty$ is no more than $e$. The order of growth of $(w I-P)_{i, i_{2}}(w I-P)_{p, i_{4}}\left[V_{\zeta\left(i_{4}, k_{1}\right)}, V_{\zeta\left(i_{2}, k_{2}\right)}\right] \bullet \psi$ at $\infty$ is no more than the order of growth of $\left[V_{\zeta\left(i_{4}, k_{1}\right)}, V_{\zeta\left(i_{2}, k_{2}\right)}\right] \bullet \psi$ at $\infty$ if $i_{2} \neq i$ and $i_{4} \neq p$, no more than 1 greater if either $i_{2}=i$ or $i_{4}=p$ but not both, and exactly 2 greater if $i_{2}=i$ and $i_{4}=p$. In interpreting this statement, zero terms must be discarded, but they make no contribution to the sum in any case. It follows that the order of growth of the left-hand side at $\infty$ is exactly $e+2$. This is a contradiction and it follows that we must have $\left[V_{\zeta}, V_{\xi}\right]=0$ for all $\zeta, \xi \in R_{13}$, as required.

Recall that $z \notin z_{0}+\sigma(F)$ is a blanket assumption in this section. We emphasize it in the next result for clarity of reference.

THEOREM 6.5. Let $\psi \in \mathbf{C} \llbracket x_{\mu}, x_{v} \rrbracket$ be a formal power series and suppose that $z \notin z_{0}+\sigma(F)$. Then the $\Omega^{(z)}$-system has one and only one solution $\Phi \in \mathbf{C} \llbracket x_{\mu}, x_{v}, x_{\zeta} \rrbracket$ such that $\Phi\left(x_{\mu}, x_{v}, 0\right)=\psi\left(x_{\mu}, x_{v}\right)$. In fact,

$$
\Phi=\prod_{\zeta \in R_{13}} \exp \left(x_{\zeta} V_{\zeta}\right) \bullet \psi
$$

If $\psi$ is a polynomial then so is $\Phi$ and, moreover, we have $\operatorname{deg}_{R_{13}}(\Phi) \leq \frac{1}{2} \operatorname{deg}(\psi)$, where $\operatorname{deg}_{R_{13}}$ denotes the degree that arises from assigning each $x_{\zeta}$ degree 1 and the remaining variables degree 0 , and deg denotes the usual degree.

Proof. Since the operator $F$ is degree preserving, its spectrum on formal power series coincides with its spectrum on polynomials. For the same reason, 
the fact that the operators $V_{\zeta}$ commute with one another in their action on polynomials implies that they commute in their action on formal power series. The facts that the IVP for the $\Omega^{(z)}$-system has one and only one solution and that the solution is given by the indicated formula now follow immediately, as they would for any system in evolutionary form with no non-trivial integrability conditions. For the remaining statement, it suffices to observe that we have $\operatorname{deg}\left(V_{\zeta} \bullet q\right) \leq \operatorname{deg}(q)-2$ for any polynomial $q$. Thus $\Phi$ cannot contain any term whose total degree in the $x_{\zeta}$-variables exceeds $\frac{1}{2} \operatorname{deg}(\psi)$.

Corollary 6.6. Let $\psi \in \mathbf{C} \llbracket x_{\mu}, x_{v} \rrbracket$ be a formal power series and suppose that $z \notin z_{0}+\sigma(F)$. Let $\Phi\left(x_{\mu}, x_{v}, x_{\zeta}\right)$ be the solution to the $\Omega^{(z)}$-system with $\Phi\left(x_{\mu}, x_{v}, 0\right)=\psi\left(x_{\mu}, x_{v}\right)$. Then

$$
\Phi_{0}\left(x_{\mu}, x_{v}, x_{\zeta}\right)=\lim _{w \rightarrow \infty} \Phi\left(x_{\mu}, x_{v}, w x_{\zeta}\right)
$$

exists and is the unique solution to the system

$$
\frac{\partial \Phi_{0}}{\partial x_{\zeta}}=\Delta_{\zeta} \bullet \Phi_{0} \quad\left(\zeta \in R_{13}\right)
$$

such that $\Phi_{0}\left(x_{\mu}, x_{v}, 0\right)=\psi\left(x_{\mu}, x_{v}\right)$.

Proof. Given the local finiteness of the operators involved, so that no convergence issues arise, this is a consequence of the expression for $\Phi$ given in Theorem 6.5 and the observation that

$$
\lim _{w \rightarrow \infty} w V_{\zeta}=\Delta_{\zeta}
$$

for all $\zeta \in R_{13}$.

\section{Umeda's Adjoint Identity and the $V$-Operators}

The $V$-operators that were introduced in Section 6 are, unfortunately, awkward to compute with in most situations. In this section, we explain how a non-commutative adjoint identity due to Umeda [9] or, more exactly, the proof of this identity, yields a method for computing the action of the $V$ operators on polynomials. We continue with the notation and assumptions introduced in Section 6. In particular, we assume that $w \notin \sigma(F)$.

We begin by introducing some objects and facts associated with Umeda's identity. Note that we require normalizations different from those that Umeda 
used. The normalizations used here are the same as those that were used in [7, Subsection 5.1], but the notation has been harmonized. It follows from Lemma 3.1 that the operators $P_{i, p}$ span a Lie algebra isomorphic to $\operatorname{gl}(a)$. Note, however, that the isomorphism $P_{i, p} \mapsto E_{p, i} \in \mathfrak{g l}(a)$ involves an interchange of indices. This circumstance accounts for the need to change the normalizations. Let

$$
\tilde{P}(w)=\left[P_{m, n}-\delta_{m n}(w+a-m)\right] .
$$

Let $e_{1}, \ldots, e_{a}$ be the standard basis for $\mathbf{C}^{a}$ and define

$$
\tau_{q}(w)=\sum_{p=1}^{a}\left(P_{p, q}-\delta_{p q} w\right) e_{p} .
$$

The first observation on the path to Umeda's formula is that we have

$$
\tau_{1}(w+a-1) \wedge \tau_{2}(w+a-2) \wedge \cdots \wedge \tau_{a}(w)=\operatorname{cdet}(\tilde{P}(w)) e_{1} \wedge \cdots \wedge e_{a},
$$

where

$$
\operatorname{cdet}(A)=\sum_{\pi} \operatorname{sgn}(\pi) A_{\pi(1) 1} \cdots A_{\pi(a) a}
$$

is the column determinant of the matrix $A$.

In $\operatorname{gl}(a)$, we use the Cartan subalgebra spanned by $P_{1,1}, \ldots, P_{a, a}$ and the positive system such that $P_{i, p}$ is a positive root vector if $i<p$. Note that, in light of the interchange of indices noted above, this choice corresponds to the opposite of the usual positive system in $\mathfrak{g l}(a)$. In particular, the highest weight of a finitedimensional irreducible $\mathrm{gl}(a)$-module will have the form $\varpi=\left(\varpi_{1}, \ldots, \varpi_{a}\right)$ with $\varpi_{1} \leq \varpi_{2} \leq \cdots \leq \varpi_{a}$. It emerges that $\operatorname{cdet}(\tilde{P}(w))$ acts on the irreducible module with highest weight $\varpi$ via the scalar

$$
c(w, \varpi)=\left(\varpi_{1}-(w+a-1)\right)\left(\varpi_{2}-(w+a-2)\right) \cdots\left(\varpi_{a}-w\right) .
$$

Next we define

$$
\begin{aligned}
\eta_{1}(w)= & (-1)^{a-1} \tau_{2}(w+a-1) \wedge \cdots \wedge \tau_{a}(w+1), \\
\eta_{m}(w)= & (-1)^{a-m} \tau_{1}(w+a-1) \wedge \cdots \wedge \tau_{m-1}(w+a-m+1) \\
& \wedge \tau_{m+1}(w+a-m) \wedge \cdots \wedge \tau_{a}(w+1), \\
\eta_{a}(w)= & \tau_{1}(w+a-1) \wedge \cdots \wedge \tau_{a-1}(w+1),
\end{aligned}
$$


where $2 \leq m \leq a-1$. These forms may be expressed as

$$
\eta_{m}(w)=\sum_{i=1}^{a}(-1)^{a-i} T_{m, i}(w) e_{1} \wedge \cdots \wedge \widehat{e}_{i} \wedge \cdots \wedge e_{a}
$$

for $1 \leq m \leq a$, where the hat denotes omission. From this we obtain an $a$-by- $a$ matrix $T(w)$. Umeda's identity [9, Proposition 2] states that

$$
T(w)(P-w I)=\operatorname{cdet}(\tilde{P}(w)) I_{a} .
$$

A proof of this identity with our normalizations is sketched in [7, Lemma 5.3].

If $\psi$ is a vector in a finite-dimensional $\mathfrak{g l}(a)$-representation then there is a canonical decomposition

$$
\psi=\sum_{\varpi} \psi_{\varpi}
$$

where $\psi_{\varpi}$ denotes the component of $\psi$ in the isotypic submodule for the irreducible representation with highest weight $\varpi$. If $1 \leq k \leq a$ and $\psi \in \mathbf{C}\left[x_{\mu}, x_{v}\right]$ then we define

$$
v_{k}(\psi)=\sum_{i=1}^{a}\left(\Delta_{\zeta(i, k)} \bullet \psi\right) e_{i} \in \mathbf{C}\left[x_{\mu}, x_{v}\right] \otimes \mathbf{C}^{a} .
$$

Suppose that $\eta$ is an $m$-form on $\mathbf{C}^{a}$ with operator coefficients and $v$ is an $n$-form on $\mathbf{C}^{a}$ with polynomial coefficients. Then we write $\eta \dot{\wedge} v$ for the $(m+n)$-form on $\mathbf{C}^{a}$ with polynomial coefficients that is obtained by taking the exterior product of the two forms while applying the operator coefficients to the polynomial coefficients. The bullet above the wedge is intended to emphasize the latter operation. With this notation in place, we define $S_{\zeta(m, k)}(w, \psi) \in \mathbf{C}\left[w, x_{\mu}, x_{v}\right]$ by

$$
\eta_{m}(w) \stackrel{\wedge}{ } v_{k}(\psi)=S_{\zeta(m, k)}(w, \psi) e_{1} \wedge \cdots \wedge e_{a}
$$

for $1 \leq m \leq a, 1 \leq k \leq c$, and $\psi \in \mathbf{C}\left[x_{\mu}, x_{v}\right]$

Proposition 7.1. For $\psi \in \mathbf{C}\left[x_{\mu}, x_{v}\right]$ we have

$$
V_{\zeta} \bullet \psi=-\sum_{\varpi} \frac{1}{c(w, \varpi)}\left(S_{\zeta}(w, \psi)\right)_{\varpi}
$$

for all $\zeta \in R_{13}$. 
Proof. We have

$$
\begin{aligned}
S_{\zeta(m, k)}(w, \psi) e_{1} \wedge \cdots \wedge e_{a} & =\eta_{m}(w) \dot{\wedge} v_{k}(\psi) \\
& =\sum_{i=1}^{a}(-1)^{a-i} T_{m, i}(w) e_{1} \wedge \cdots \wedge \widehat{e_{i}} \wedge \cdots \wedge e_{a} \wedge v_{k}(\psi) \\
& =\sum_{i=1}^{a}(-1)^{a-i} T_{m, i}(w) e_{1} \wedge \cdots \wedge \widehat{e}_{i} \wedge \cdots \wedge e_{a} \wedge\left(\Delta_{\zeta(i, k)} \bullet \psi\right) e_{i} \\
& =\left(\sum_{i=1}^{a} T_{m, i}(w) \Delta_{\zeta(i, k)} \bullet \psi\right) e_{1} \wedge \cdots \wedge e_{a}
\end{aligned}
$$

and so

$$
S_{\zeta(m, k)}(w, \psi)=\sum_{i=1}^{a} T_{m, i}(w) \Delta_{\zeta(i, k)} \bullet \psi
$$

It follows that

$$
\begin{aligned}
S_{\zeta(m, k)}(w, \psi) & =\sum_{i_{1}=1}^{a} T_{m, i_{1}}(w) \Delta_{\zeta\left(i_{1}, k\right)} \bullet \psi \\
& =\sum_{i_{1}, i_{2}=1}^{a} T_{m, i_{1}}(w)(w I-P)_{i_{1}, i_{2}} V_{\zeta\left(i_{2}, k\right)} \bullet \psi \\
& =-\sum_{i_{2}=1}^{a} \operatorname{cdet}(\tilde{P}(w)) \delta_{m i_{2}} V_{\zeta\left(i_{2}, k\right)} \bullet \psi \\
& =-\operatorname{cdet}(\tilde{P}(w)) V_{\zeta(m, k)} \bullet \psi,
\end{aligned}
$$

by Umeda's adjoint identity. In turn, this gives

$$
\begin{aligned}
S_{\zeta(m, k)}(w, \psi) & =-\sum_{\varpi} \operatorname{cdet}(\tilde{P}(w))\left(V_{\zeta(m, k)} \bullet \psi\right)_{\varpi} \\
& =-\sum_{\varpi} c(w, \varpi)\left(V_{\zeta(m, k)} \bullet \psi\right)_{\varpi}
\end{aligned}
$$

and so

$$
\left(S_{\zeta(m, k)}(w, \psi)\right)_{\varpi}=-c(w, \varpi)\left(V_{\zeta(m, k)} \bullet \psi\right)_{\varpi} .
$$

This is equivalent to the stated identity. 
We can refine the estimate on the $R_{13}$-degree of $\Phi$ given in Theorem 6.5 by recalling some further results about the structure of the space $\mathbf{C}\left[x_{\mu}, x_{v}\right]$. Indeed, there is an isomorphism

$$
\mathbf{C}\left[x_{\mu}, x_{v}\right] \cong \mathbf{C}\left[\varphi_{\zeta}\right] \otimes \mathscr{H}
$$

where $\mathbf{C}\left[\varphi_{\zeta}\right]$ denotes the algebra generated by the $\varphi_{\zeta}$ and $\mathscr{H}$ denotes the space of harmonic polynomials. The inverse isomorphism satisfies $\varphi^{\beta} \otimes h \mapsto \varphi^{\beta} h$. Thus any non-zero polynomial $\psi \in \mathbf{C}\left[x_{\mu}, x_{v}\right]$ may be expressed uniquely in the form

$$
\psi=\sum_{\beta} h_{\beta} \varphi^{\beta},
$$

where $\beta$ runs over $\mathbf{N}^{R_{13}}, h_{\beta} \in \mathscr{H}$, and $h_{\beta}=0$ for all but finitely-many values of

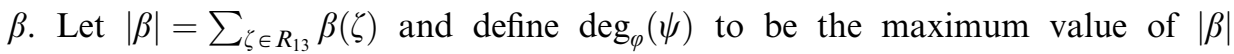
over all $\beta$ such that $h_{\beta} \neq 0$. It is convenient to extend $\operatorname{deg}_{\varphi}$ by defining $\operatorname{deg}_{\varphi}(0)=-\infty$. Since $\operatorname{deg}\left(\varphi_{\zeta}\right)=2$ for all $\zeta \in R_{13}$, we have $\operatorname{deg}_{\varphi}(\psi) \leq \frac{1}{2} \operatorname{deg}(\psi)$. For this reason, the statement of Proposition 7.3 strengthens the last statement of Theorem 6.5.

Lemma 7.2. Let $\psi \in \mathbf{C}\left[x_{\mu}, x_{v}\right]$. Then

$$
\operatorname{deg}_{\varphi}(\psi)=\max _{\varpi}\left\{\operatorname{deg}_{\varphi}\left(\psi_{\varpi}\right)\right\}
$$

Proof. Since $\psi=\sum_{\varpi} \psi_{\varpi}$, it is clear that we have

$$
\operatorname{deg}_{\varphi}(\psi) \leq \max _{\varpi}\left\{\operatorname{deg}_{\varphi}\left(\psi_{\varpi}\right)\right\}
$$

For the reverse inequality note that, by the Jacobson Density Theorem, for each $\varpi$ there is an element $u_{\varpi} \in \mathscr{U}(\mathfrak{g l}(a))$ such that $\psi_{\varpi}=u_{\varpi} \bullet \psi$. The element $u_{\varpi}$ will be a linear combination of monomials in the operators $P_{i, p}$. If $h$ is a harmonic polynomial then

$$
P_{i, p} \bullet\left(\varphi^{\beta} h\right)=\left(P_{i, p} \bullet \varphi^{\beta}\right) h+\varphi^{\beta}\left(P_{i, p} \bullet h\right) .
$$

By Lemma 3.2, $P_{i, p} \bullet h$ is harmonic. Inspection of Lemma 3.4 shows that $P_{i, p} \bullet \varphi^{\beta}$ is a linear combination of various $\varphi^{\gamma}$ with $|\gamma|=|\beta|$. These observations imply that

$$
\operatorname{deg}_{\varphi}\left(P_{i, p} \bullet\left(\varphi^{\beta} h\right)\right) \leq \operatorname{deg}_{\varphi}\left(\varphi^{\beta} h\right)
$$


It follows that $\operatorname{deg}_{\varphi}\left(P_{i, p} \bullet \psi\right) \leq \operatorname{deg}_{\varphi}(\psi)$ and hence, inductively, that

$$
\operatorname{deg}_{\varphi}\left(\psi_{\varpi}\right)=\operatorname{deg}_{\varphi}\left(u_{\varpi} \bullet \psi\right) \leq \operatorname{deg}_{\varphi}(\psi) .
$$

The required conclusion follows from this.

Proposition 7.3. Let $\psi \in \mathbf{C}\left[x_{\mu}, x_{v}\right]$ and $\Phi \in \mathbf{C}\left[x_{\mu}, x_{v}, x_{\zeta}\right]$ be the unique solution to the $\Omega$-system with $\Phi\left(x_{\mu}, x_{v}, 0\right)=\psi\left(x_{\mu}, x_{v}\right)$. Then $\operatorname{deg}_{R_{13}}(\Phi) \leq \operatorname{deg}_{\varphi}(\psi)$.

Proof. In light of Theorem 6.5, the conclusion will follow if we can show that $\operatorname{deg}_{\varphi}\left(V_{\zeta} \bullet \psi\right) \leq \operatorname{deg}_{\varphi}(\psi)-1$ for all $\zeta \in R_{13}$. First consider $\Delta_{\zeta} \bullet\left(\varphi^{\beta} h\right)$ with $h$ a harmonic polynomial. Inspection of Proposition 3.5 reveals that

$$
\operatorname{deg}_{\varphi}\left(\Delta_{\zeta} \bullet\left(\varphi^{\beta} h\right)\right) \leq \operatorname{deg}_{\varphi}\left(\varphi^{\beta} h\right)-1
$$

and it follows that $\operatorname{deg}_{\varphi}\left(\Delta_{\zeta} \bullet \psi\right) \leq \operatorname{deg}_{\varphi}(\psi)-1$ for all $\psi$. Now

$$
S_{\zeta(m, k)}(w, \psi)=\sum_{i=1}^{a} T_{m, i}(w) \Delta_{\zeta(i, k)} \bullet \psi
$$

with $T_{m, i}(w) \in \mathbf{C}[w] \otimes \mathscr{U}(\mathfrak{g l}(a))$. It follows from this and the observation made in the proof of Lemma 7.2 that $\operatorname{deg}_{\varphi}\left(S_{\zeta}(w, \psi)\right) \leq \operatorname{deg}_{\varphi}(\psi)-1$ for all $\zeta \in R_{13}$. Lemma 7.2 itself then $\operatorname{gives}^{\operatorname{deg}_{\varphi}}\left(S_{\zeta}(w, \psi)_{\varpi}\right) \leq \operatorname{deg}_{\varphi}(\psi)-1$ for all $\zeta \in R_{13}$ and all weights $\varpi$. Now we apply Proposition 7.1 to conclude that $\operatorname{deg}_{\varphi}\left(V_{\zeta} \bullet \psi\right) \leq \operatorname{deg}_{\varphi}(\psi)-1$ for all $\zeta \in R_{13}$, as required.

\section{Examples of Radial Solutions}

We call a solution $\Phi\left(x_{\mu}, x_{v}, x_{\zeta}\right) \in \mathbf{C}\left[x_{\mu}, x_{v}, x_{\zeta}\right]$ to the $\Omega$-system radial if $\Phi\left(x_{\mu}, x_{v}, 0\right) \in \mathbf{C}[\varphi]$. The purpose of this section is to illustrate some of the results found above by giving the radial solutions to the $\Omega$-system explicitly in two of the three cases in which this is presently possible. The cases we deal with here are those where $a=1$ or $c=1$. The third case, where $b=1$, will be dealt with elsewhere, since it requires additional methods and has a number of other interesting features.

We continue with the notation that was introduced in previous sections; in particular, $w=z-z_{0}$. We also continue to assume that $w \notin \sigma(F)$. We recall the Lauricella hypergeometric series of type $\mathrm{D}$, which is defined by

$$
F_{D}\left(\alpha, \beta_{1}, \ldots, \beta_{n}, \gamma ; u_{1}, \ldots, u_{n}\right)=\sum_{m} \frac{(\alpha)_{m_{+}}\left(\beta_{1}\right)_{m_{1}} \cdots\left(\beta_{n}\right)_{m_{n}}}{(\gamma)_{m_{+}} m_{1} ! \cdots m_{n} !} u^{m}
$$


In this expression, the sum is over $\mathbf{N}^{n},(\cdot)_{m}$ is the rising factorial (which is defined inductively by $(\varepsilon)_{0}=1$ and $(\varepsilon)_{m}=(\varepsilon)_{m-1}(\varepsilon+m-1)$ for $\left.m \geq 1\right), u^{m}$ is shorthand for $u_{1}^{m_{1}} \cdots u_{n}^{m_{n}}$, and $m_{+}$is shorthand for $m_{1}+\cdots+m_{n}$. It is well known that this series converges at least when $\left|u_{i}\right|<1$ for $1 \leq i \leq n$. Note that if $\beta_{i} \in-\mathbf{N}$ for $1 \leq i \leq n$ then the series has only finitely-many non-zero terms.

First, we consider the case where $a=1$. Under this assumption, the first index in $\mu(i, j)$ and in $\zeta(i, k)$ becomes redundant and so we drop it. Thus we write

$$
\varphi^{\beta}=\varphi_{\zeta(1)}^{\beta_{1}} \cdots \varphi_{\zeta(c)}^{\beta_{c}},
$$

where

$$
\varphi_{\zeta(k)}=\sum_{j=1}^{b} x_{\mu(j)} x_{v(j, k)}
$$

THeOREM 8.1. Suppose that $a=1$ and that $\beta \in \mathbf{N}^{c}$. Then the unique solution $\Phi$ to the $\Omega^{(z)}$-system with $\Phi\left(x_{\mu}, x_{v}, 0\right)=\varphi^{\beta}$ is given by

$$
\varphi^{\beta} F_{D}\left(1-b-\beta_{+},-\beta_{1}, \ldots,-\beta_{c}, w-\beta_{+}+1 ; \frac{x_{\zeta(1)}}{\varphi_{\zeta(1)}}, \ldots, \frac{x_{\zeta(c)}}{\varphi_{\zeta(c)}}\right)
$$

where $\beta_{+}=\beta_{1}+\cdots+\beta_{c}$.

Proof. There are $c$ of the $\Delta$-operators in this case, given by

$$
\Delta_{\zeta(k)}=\sum_{j=1}^{b} \frac{\partial^{2}}{\partial x_{\mu(j)} \partial x_{v(j, k)}}
$$

for $1 \leq k \leq c$. It follows from Proposition 3.5 that

$$
\Delta_{\zeta(k)} \bullet \varphi^{\beta}=\beta_{k}\left(\beta_{+}+b-1\right) \varphi^{\beta-e_{k}},
$$

where $e_{k}$ is 1 in the $k^{\text {th }}$ position and 0 elsewhere. There is a single $P$-operator in this case and it is $\mathbf{E}_{\mu}$, the Euler operator in the variables $x_{\mu(1)}, \ldots, x_{\mu(c)}$. Thus $V_{\zeta(k)}=\left(w-\mathbf{E}_{\mu}\right)^{-1} \Delta_{\zeta(k)}$ and this leads to

$$
V_{\zeta(k)} \bullet \varphi^{\beta}=\frac{\beta_{k}\left(\beta_{+}+b-1\right)}{w-\beta_{+}+1} \varphi^{\beta-e_{k}},
$$

which we prefer to write as

$$
V_{\zeta(k)} \bullet \varphi^{\beta}=\frac{\left(-\beta_{k}\right)\left(1-b-\beta_{+}\right)}{w-\beta_{+}+1} \varphi^{\beta-e_{k}}
$$


so that we may use rising, rather than falling, factorials at the next step. Indeed, the previous identity gives

$$
V_{\zeta(k)}^{m} \bullet \varphi^{\beta}=\frac{\left(-\beta_{k}\right)_{m}\left(1-b-\beta_{+}\right)_{m}}{\left(w-\beta_{+}+1\right)_{m}} \varphi^{\beta-m e_{k}}
$$

for $m \geq 0$ and so

$$
\exp \left(x_{\zeta(k)} V_{\zeta(k)}\right) \bullet \varphi^{\beta}=\sum_{m=0}^{\infty} \frac{\left(-\beta_{k}\right)_{m}\left(1-b-\beta_{+}\right)_{m}}{\left(w-\beta_{+}+1\right)_{m} m !} \varphi^{\beta-m e_{k}} x_{\zeta(k)}^{m} .
$$

Now suppose that $1 \leq r, k \leq c$ with $r \neq k$. Then

$$
\begin{aligned}
& \exp \left(x_{\zeta(r)} V_{\zeta(r)}\right) \exp \left(x_{\zeta(k)} V_{\zeta(k)}\right) \bullet \varphi^{\beta} \\
&=\sum_{m_{k}=0}^{\infty} \frac{\left(-\beta_{k}\right)_{m_{k}}\left(1-b-\beta_{+}\right)_{m_{k}}}{\left(w-\beta_{+}+1\right)_{m_{k}} m_{k} !} x_{\zeta(k)}^{m_{k}} \exp \left(x_{\zeta(r)} V_{\zeta(r)}\right) \bullet \varphi^{\beta-m_{k} e_{k}} \\
&=\sum_{m_{k}=0}^{\infty} \sum_{m_{r}=0}^{\infty} \frac{\left(-\beta_{k}\right)_{m_{k}}\left(1-b-\beta_{+}\right)_{m_{k}}}{\left(w-\beta_{+}+1\right)_{m_{k}} m_{k} !} x_{\zeta(k)}^{m_{k}} \\
& \cdot \frac{\left(-\beta_{r}\right)_{m_{r}}\left(1-b-\beta_{+}+m_{k}\right)_{m_{r}}}{\left(w-\beta_{+}+1+m_{k}\right)_{m_{r}} m_{r} !} x_{\zeta(r)}^{m_{r}} \varphi^{\beta-m_{k} e_{k}-m_{r} e_{r}} \\
&= \sum_{m_{k}=0}^{\infty} \sum_{m_{r}=0}^{\infty} \frac{\left(-\beta_{k}\right)_{m_{k}}\left(-\beta_{r}\right)_{m_{r}}\left(1-b-\beta_{+}\right)_{m_{k}+m_{r}}}{\left(w-\beta_{+}+1\right)_{m_{k}+m_{r}} m_{k} ! m_{r} !} x_{\zeta(k)}^{m_{k}} x_{\zeta(r)}^{m_{r}} \varphi^{\beta-m_{k} e_{k}-m_{r} e_{r}} .
\end{aligned}
$$

In this computation, we have used the elementary identity $(\varepsilon)_{m}(\varepsilon+m)_{n}=(\varepsilon)_{m+n}$. By proceeding in this way, we obtain

$$
\prod_{k=1}^{c} \exp \left(x_{\zeta(k)} V_{\zeta(k)}\right) \bullet \varphi^{\beta}=\sum_{m} \frac{\left(-\beta_{1}\right)_{m_{1}} \cdots\left(-\beta_{c}\right)_{m_{c}}\left(1-b-\beta_{+}\right)_{m_{+}}}{\left(w-\beta_{+}+1\right)_{m_{+}} m_{1} ! \cdots m_{c} !} x_{\zeta}^{m} \varphi^{\beta-m}
$$

where $x_{\zeta}^{m}$ is shorthand for $x_{\zeta(1)}^{m_{1}} \cdots x_{\zeta(c)}^{m_{c}}$. This is equivalent to the stated formula.

Note that the $R_{13}$-degree of the solution given in Theorem 8.1 is exactly $\beta_{+}=\operatorname{deg}_{\varphi}\left(\varphi^{\beta}\right)$ and so the inequality of Proposition 7.3 is an equality in this case. 
Lemma 8.2. Suppose that $\beta \in \mathbf{N}^{n}$ and $\gamma \notin 1-\beta_{+}+\mathbf{N}$. Then we have

$$
F_{D}\left(\alpha,-\beta_{1}, \ldots,-\beta_{n}, \gamma ; 1, \ldots, 1\right)=\frac{(\gamma-\alpha)_{\beta_{+}}}{(\gamma)_{\beta_{+}}}
$$

Proof. This is an analogue of the terminating form of Gauss' evaluation of the Gaussian hypergeometric function at 1 (which is equivalent to the ChuVandermonde identity) and may be proved in the same ways. One is to begin with the Eulerian integral

$$
\begin{aligned}
& F_{D}\left(\alpha,-\beta_{1}, \ldots,-\beta_{n}, \gamma ; u_{1}, \ldots, u_{n}\right) \\
& \quad=\frac{\Gamma(\gamma)}{\Gamma(\alpha) \Gamma(\gamma-\alpha)} \int_{0}^{1} t^{\alpha-1}(1-t)^{\gamma-\alpha-1}\left(1-u_{1} t\right)^{\beta_{1}} \cdots\left(1-u_{n} t\right)^{\beta_{n}} d t
\end{aligned}
$$

valid for all $u_{1}, \ldots, u_{n}$ under the assumption on $\beta_{1}, \ldots, \beta_{n}$ made in the statement and provided that $\operatorname{re}(\gamma)>\operatorname{re}(\alpha)>0$. We substitute $u_{1}=\cdots=u_{n}=1$, evaluate the resulting beta integral, and then observe that both sides are rational functions of $\alpha$ and $\gamma$ so that the identity extends to all $\alpha$ and $\gamma$ for which the denominator does not vanish.

We next consider the case where $c=1$. In this case, the second index in $v(j, k)$ and in $\zeta(i, k)$ becomes redundant and so we drop it. Thus we write

$$
\varphi^{\beta}=\varphi_{\zeta(1)}^{\beta_{1}} \cdots \varphi_{\zeta(a)}^{\beta_{a}},
$$

where

$$
\varphi_{\zeta(i)}=\sum_{j=1}^{b} x_{\mu(i, j)} x_{v(j)} .
$$

Theorem 8.3. Suppose that $c=1$, that $\beta \in \mathbf{N}^{a}$, and that $w \notin \mathbf{Z}$. Then the unique solution to the $\Omega^{(z)}$-system that satisfies $\Phi\left(x_{\mu}, x_{v}, 0\right)=\varphi^{\beta}$ is given by

$$
\begin{gathered}
\frac{\left(1-b-\beta_{+}-w\right)_{\beta_{+}}}{(-w)_{\beta_{+}}} \varphi^{\beta} F_{D}\left(1-b-\beta_{+},-\beta_{1}, \ldots,-\beta_{a}, 1-b-\beta_{+}-w ;\right. \\
\left.1-\frac{x_{\zeta(1)}}{\varphi_{\zeta(1)}}, \ldots, 1-\frac{x_{\zeta(a)}}{\varphi_{\zeta(a)}}\right) .
\end{gathered}
$$


Proof. We use the notion of conjugate $\Omega$-systems that was introduced in Section 5. An $\Omega$-system with $c=1$ is conjugate to an $\Omega$-system with $a=1$. The transformation from the original system to the conjugate system is given by the substitutions

$$
\begin{aligned}
& x_{v(j)} \rightsquigarrow x_{\mu(j)}, \\
& x_{\mu(i, j)} \rightsquigarrow x_{v(j, i)}, \\
& x_{\zeta(i)} \rightsquigarrow \varphi_{\zeta(i)}-x_{\zeta(i)}, \\
& z \rightsquigarrow-z
\end{aligned}
$$

and these substitutions entail

$$
\begin{gathered}
\varphi_{\zeta(i)} \rightsquigarrow \varphi_{\zeta(i)}, \\
w \leadsto-b-w .
\end{gathered}
$$

Note that the assumption that $w \notin \mathbf{Z}$ implies that $-b-w \notin \sigma(F)$ since $\sigma(F) \subset \mathbf{Z}$. It follows from Proposition 5.1 and Theorem 8.1 that

$$
\varphi^{\beta} F_{D}\left(1-b-\beta_{+},-\beta_{1}, \ldots,-\beta_{a}, 1-b-\beta_{+}-w ; 1-\frac{x_{\zeta(1)}}{\varphi_{\zeta(1)}}, \ldots, 1-\frac{x_{\zeta(a)}}{\varphi_{\zeta(a)}}\right)
$$

is a solution to $\Omega$-system that we are considering. On the set where $x_{\zeta(i)}=0$ for $1 \leq i \leq a$ this function takes the value

$$
\varphi^{\beta} F_{D}\left(1-b-\beta_{+},-\beta_{1}, \ldots,-\beta_{a}, 1-b-\beta_{+}-w ; 1, \ldots, 1\right)
$$

and, by Lemma 8.2 , this is equal to

$$
\frac{(-w)_{\beta_{+}}}{\left(1-b-\beta_{+}-w\right)_{\beta_{+}}} \varphi^{\beta} \text {. }
$$

Note that the hypothesis that $w \notin \mathbf{Z}$ is used again here, and also implies that $(-w)_{\beta_{+}} \neq 0$. It follows that we may normalize the above solution by multiplying it by $\left(1-b-\beta_{+}-w\right)_{\beta_{+}} /(-w)_{\beta_{+}}$to obtain the required restriction to the set where $x_{\zeta(i)}=0$ for $1 \leq i \leq a$.

In the case of the Heisenberg ultrahyperbolic equation both $a$ and $c$ are 1 . Here, Lauricella's function $F_{D}$ reduces to the Gaussian hypergeometric function and the solutions given by Theorems 8.1 and 8.3 are related by a transformation due to Pfaff. The reader may consult the second formula given in [1, Corollary 2.3.3] for this transformation. 


\section{References}

[1] G. Andrews, R. Askey, and R. Roy, Special Functions, Encyclopedia of Mathematics and Its Applications, vol. 71, Cambridge University Press, Cambridge, 1999.

[2] G. B. Folland and E. M. Stein, Estimates for the $\bar{\partial}_{b}$ complex and analysis on the Heisenberg group, Comm. Pure Appl. Math. 27 (1974), 429-522.

[3] P. C. Greiner and T. H. Koornwinder, Variations on the Heisenberg spherical harmonics. Report ZW 186/83. Mathematisch Centrum, Amsterdam, 1983.

[4] F. John, The ultrahyperbolic equation with 4 independent variables, Duke Math. J. 4 (1938), 300-322.

[5] A. C. Kable, The Heisenberg ultrahyperbolic equation: the basic solutions as distributions, Pacific J. Math. 258 (2012), 165-197.

[6] A. C. Kable, The Heisenberg ultrahyperbolic equation: $K$-finite and polynomial solutions, Kyoto J. Math. 52 (2012), no. 4, 839-894.

[7] A. C. Kable, On certain conformally invariant systems of differential equations, New York J. Math. 19 (2013), 189-251.

[ 8 ] A. Korányi, Kelvin transforms and harmonic polynomials on the Heisenberg group, J. Funct. Anal. 49 (1982), 177-185.

[ 9 ] T. Umeda, Newton's formula for $\mathrm{gl}_{n}$, Proc. Amer. Math. Soc. 126 (1998), no. 11, 3169-3175.

Department of Mathematics

Oklahoma State University

Stillwater OK 74078, USA

E-mail: akable@math.okstate.edu 